\title{
NILPOTENT STRUCTURES AND INVARIANT METRICS ON COLLAPSED MANIFOLDS
}

\author{
JEFF CHEEGER, KENJI FUKAYA, AND MIKHAEL GROMOV
}

\section{CONTENTS}

I. Introduction

0 . Background

1. Statement of main results and outline of their proof

II. Preliminaries

2. Smoothing Hausdorff approximations

3. Equivariant and parameterized version of the theorem on almost flat manifolds

4. Nilpotent Killing structures on fibrations

III. The nilpotent Killing structure and invariant round metric

5. Local fibration of the frame bundle

6. Making the local fibrations compatible

7. Making the local group actions compatible

8. The induced structure and metric on the base

Appendix 1. Local structure of Riemannian manifolds of bounded curvature Appendix 2. Fibration isotopy

\section{INTRODUCTION}

\section{BACKGROUND}

Let $M^{n}$ be a complete Riemannian manifold of bounded curvature, say $|K| \leq 1$. Given a small number, $\varepsilon>0$, we put $M^{n}=\mathscr{B}^{n}(\varepsilon) \cup \mathscr{C}^{n}(\varepsilon)$, where $\mathscr{B}^{n}(\varepsilon)$ consists of those points at which the injectivity radius of the exponential map is $\geq \varepsilon$. The complementary set, $\mathscr{C}^{n}(\varepsilon)$ is called the $\varepsilon$-collapsed part of $M^{n}$.

If $x \in \mathscr{B}^{n}(\varepsilon), r \leq \varepsilon$, then the metric ball $B_{x}(r)$ is quasi-isometric, with small distortion, to the flat ball $B_{0}(r)$ in the Euclidean space, $R^{n}$. After slightly

Received by the editors May 15, 1991.

1991 Mathematics Subject Classification. Primary 53C20.

The first author was partially supported by NSF Grant DMS 840596 . 
adjusting the boundary of $\mathscr{B}^{n}(\varepsilon)$, we obtain a set whose quasi-isometry type is determined up to a finite number of possibilities by the ratio, $\operatorname{dia}\left(\mathscr{B}^{n}(\varepsilon)\right) / \varepsilon$, where $\operatorname{dia}\left(\mathscr{B}^{n}(\varepsilon)\right)$ denotes the diameter of $\mathscr{B}^{n}(\varepsilon)$. (Compare [C, GLP, GW, P]).

In this paper, we are concerned with what can be said about the $\varepsilon$-collapsed part, $\mathscr{C}^{n}(\varepsilon)$, for $\varepsilon=\varepsilon(n)$ a suitably small constant depending only on $n$. Roughly speaking, our main results show that the essential features of the local geometry are encoded in the symmetry structure of a nearby metric. More precisely, any metric of bounded curvature on $M^{n}$ can be closely approximated by one that admits a sheaf of nilpotent Lie algebras of local Killing vector fields that point in all sufficiently collapsed directions of $C^{n}(\varepsilon)$. This sheaf is called the nilpotent Killing structure.

A second sheaf of nilpotent Lie algebras of vector fields, called the nilpotent collapsing structure will be discussed elsewhere. It plays a crucial role in constructions, which collapse away all sufficiently collapsed directions in the manifold (while keeping its curvature bounded). The fact that two different sheaves arise simply reflects the distinction between right and left invariant vector fields on a nilpotent Lie group (compare Example 1.6 and the discussion preceding it).

The first nontrivial example of a collapsing sequence of Riemannian manifolds was pointed out by Marcel Berger in about 1962. Berger started with the Hopf fibration, $S^{1} \rightarrow S^{3} \rightarrow S^{2}$, where $S^{3}$ carries its standard metric. He observed that if one multiplies the lengths of the fibres by $\varepsilon$, while leaving the metric in the orthogonal directions unchanged, then the sectional curvature stays bounded independent of $\varepsilon$, as $\varepsilon \rightarrow 0$. But as $\varepsilon \rightarrow 0, S^{3}$ more and more closely resembles $S^{2}$ (equipped with a metric of constant curvature 4 ). In the process, the injectivity radius converges to zero everywhere.

The first theorem on collapse characterizes "almost flat manifolds" [G1]. These manifolds, $X^{n}$, have bounded curvature, say $|K| \leq 1$, and are collapsed in the strongest sense possible. Namely, the diameter satisfies, dia $\left(X^{n}\right) \leq \varepsilon(n)$. The theorem asserts that a finite normal covering space, $\widetilde{X}^{n}$, is diffeomorphic to a nilmanifold, $\Lambda \backslash N$.

Subsequently, by employing additional analytic arguments, Ruh proved that $X^{n}$ itself is infranil [R]. This means that the covering group of $\widetilde{X}^{n} \rightarrow X$ acts by affine transformations with respect to the canonical flat affine connection on the tangent bundle of $\widetilde{X}^{n}$. Otherwise, put $X^{n}$ is diffeomorphic to $\Lambda \backslash N$, where the covering group, $\Lambda$, acts by affine transformations, with respect to the canonical connection on $N$ and the image of the holonomy homomorphism is finite. By the canonical connection, we mean the one for which all left invariant vector fields are parallel. An important by-product of Ruh's proof is the statement that the diffeomorphism between $X^{n}$ and $\Lambda \backslash N$ can be chosen canonically, given the geometry of $X^{n}$ and a choice of base point, $x \in X^{n}$. Moreover, in this case, a canonical left invariant metric on $N$ that is actually invariant under $\Lambda$ can also be chosen.

It is easy to see that although most infranil manifolds admit no flat metric, any such manifold admits a sequence of metrics with $|K| \leq 1$, for which the 
diameter becomes arbitrarily small; see [G1]. Thus, the above-mentioned results imply the existence of a critical diameter; if $X^{n}$ admits a metric with $|K| \leq 1$ and $\operatorname{dia}\left(X^{n}\right) \leq \varepsilon(n)$, then it admits a sequence of metrics with $|K| \leq 1$ and $\operatorname{dia}\left(X^{n}\right) \rightarrow 0$.

The case of infranil manifolds illustrates a basic point. Collapse can take place simultaneously on several different length scales and not just on the scale of the injectivity radius. Indeed, the simplest nonflat nilmanifolds (with almost flat metrics) can be viewed as the total spaces of a nontrivial circle bundles, whose base spaces are isometric products of two circles of length $\varepsilon$ and whose fibres have length $\varepsilon^{2}$. This kind of inhomogeneous scaling is actually essential, in order for the curvature to remain bounded as $\varepsilon \rightarrow 0$.

The ideas on almost flat manifolds were extended along two rather different lines, in order to study the collapsing phenomenon in greater generality. The goal of the present paper is to combine these two approaches.

In [CG3, CG4], generalizing the concept of a group action, the notion of an action of a sheaf of groups was introduced. An $F$-structure is an action of a sheaf of tori for which certain additional regularity conditions hold (" $F$ " stands for "flat"). As in the case of a group action, an action of a sheaf of groups induces a partition of the underlying space into orbits. The main result of [CG4] asserts the existence of an $F$-structure of positive rank (i.e., all orbits have positive dimension) on the sufficiently collapsed part of a manifold with $|K| \leq 1$. Here, no assumption is made concerning the size of the manifold, which might even be infinite. In this generality, the dimension of the stalk of the $F$-structure is not always locally constant. If not, the structure is called mixed; if so it is called pure.

The infinitesimal generator of the local action of an $F$-structure is a sheaf of abelian Lie algebras of vector fields, which can be regarded as Killing fields for some Riemannian metric. For the $F$-structure constructed in [CG4], this metric can actually be chosen close to the original one. The Killing fields themselves point only in the "shortest" collapsed directions. As a consequence, this $F$ structure describes the local geometry of the collapsed region only on its smallest length scale, that of the injectivity radius. This accounts for the abelian (as opposed to nilpotent) character of the structure.

The existence of an $F$-structure of positive rank does impose a global constraint on the topology of the underlying space. For example, it implies that the Euler characteristic vanishes [CG3].

Example 0.1. The need to consider mixed $F$-structures in cases where diameter is not bounded is illustrated by the metric

$$
d r^{2}+e^{-(R+r)} d \theta_{1}^{2}+e^{-(R-r)} d \theta_{2}^{2},
$$

on the set $(-R, R) \times S^{1} \times S^{1},(R>>0)$. By counting the number of collapsed directions, it becomes clear that in this example, the tori that act locally near the ends are one-dimensional, while near the middle, a two-dimensional torus acts. Note however, that there is no completely canonical way of choosing the precise set of points at which the transition takes place.

There is also a converse to the existence theorem for the $F$-structure. Namely, 
associated to every $F$-structure of positive rank are sequences of metrics with $|K| \leq 1$, containing ones that are arbitrarily collapsed, for which the action of the structure is isometric (see [CG3]). This leads to the existence of a "critical injectivity radius," which is analogous to the notion of "critical diameter" as mentioned above.

In the approach due to the second author, the starting point is to consider a manifold, $M^{n}$, with $|K| \leq 1$, which, as in the Berger example above, "to the naked eye," closely resembles a lower-dimensional manifold $Y^{m}$. Technically speaking, one requires that $M^{n}$ is sufficiently close to $Y^{m}$ in the Hausdorff distance (see [GLP]). The manifold $Y^{m}$ is assumed to have bounded geometry but its diameter need not be finite. The conclusion is that there is a fibration $Z^{n-m} \rightarrow M^{n} \rightarrow Y^{m}$ whose fibre, $Z^{n-m}$, is an infranil manifold. In case $Y^{m}$ is a point, the assertion reduces to the theorem on almost flat manifolds (see [F1] and $\S 2$ of the present paper for details).

Although the context of this fibration theorem might at first seem rather special, it turns out that its equivariant generalization gives strong information on the structure of arbitrary collapsed regions of bounded diameter. The reason is as follows. Suppose that for a given manifold, both the curvature tensor and its covariant derivative are bounded (the assumption concerning the covariant derivative is actually not a serious one, since by results of [BMR], [Shi], and [A], an arbitrary metric can be approximated by one for which this holds). Then the frame bundle, $F M^{n}$, equipped with its natural metric, has bounded curvature as well. If $U^{n}$ is a region that is sufficiently collapsed relative to the size of its diameter, one can show that there exists $Y^{m}$ as in the fibration theorem, such that the frame bundle, $F U^{n}$, is sufficiently Hausdorff close to $Y^{m}$. Moreover, in this case, the fibre, $Z$, of the fibration, $Z \rightarrow F U^{n} \rightarrow Y^{m}$, is actually a nilmanifold (and not just infranil). (Ultimately, both assertions can be traced to the fact that an isometry of the base space, which fixes a point of the frame bundle, is the identity map.) The fibration, $Z^{\frac{n(n-1)}{2}+n-m} \rightarrow F U^{n} \rightarrow Y^{m}$, can be chosen to be equivariant with respect to the action of $O(n)$ on $F U^{n}$. As a consequence, a partition into infranilmanifolds, in general not all of the same dimension, is induced on $U^{n}$. These "orbits" contain all collapsed directions, and so determine all possible length scales on which collapse takes place. The flat orbits of the $F$-structure can be thought of as lying inside these nilpotent ones (in fact, they lie inside the pieces corresponding to the center of the nilpotent group); see [F3].

The fibration theorem is sharpened in another direction in [F2]. There, Ruh's theorem is used to obtain a smooth family of affine flat structures on the fibres. In fact, by Malcev's rigidity theorem, these are all affine equivalent to some fixed $\Lambda \backslash N$; see Theorem 3.7 and Proposition 3.8. As a consequence, the structural group of the fibration reduces to the group of affine automorphisms of $\Lambda \backslash N$. The existence of such a reduction is a necessary and sufficient condition for the total space of a fibration with fibre diffeomorphic to $\Lambda \backslash N$ to collapse to the base space keeping curvature bounded. Thus the theorem on the "critical diameter" generalizes to the fibration setting.

We point out that the result of [F2] is obtained without removing the dependence on the base point in Ruh's construction. As a consequence, the con- 
struction of [F2] is not $G$-equivariant. This point, which is important for the present paper, is dealt with in $\S 3$.

We refer to [CG3, CG4, F1-F3] for further background and examples.

\section{STATEMENT OF MAIN RESUltS AND OUTLINE OF THEIR PROOF}

As already mentioned, the goal of the present paper is to synthesize the two approaches to collapse that were described in the previous section. Thus, without assuming a bound on diameter, we will construct a nilpotent structure, in general of mixed type, which is nontrivial on sufficiently collapsed regions. The structure incorporates a description of the local geometry on a fixed scale and not just on the scale of the injectivity radius. It is called the nilpotent Killing structure. We will show that its action is isometric for a metric close to the original one.

As mentioned earlier, there is also a second structure, called the nilpotent collapsing structure. Although its orbits are the same as those of the nilpotent Killing structure, its construction requires a small amount of additional work. This, together with a description of its role in collapsing will be provided elsewhere; see, however, Example 1.6 and compare [F2].

The existence of a metric whose symmetry structure encodes the essential features of the geometry can be made precise without reference to sheaves. However, the compatibility between this metric and the sheaf structure imposes a consistency condition on the local symmetries at neighboring points, which captures the purely topological aspect of the discussion.

Let $(M, g)$ be a Riemannian manifold. Let $V \subset M$ be open and $\pi: \widetilde{V} \rightarrow$ $V$, a normal covering with covering group, $\Lambda$.

(1.1.1) Assume that there exists a Lie group, $H \supset \Lambda$, with finitely many components, and an isometric action of $H$ on $\widetilde{V}$, extending that of $\Lambda$, such that

(1.1.2) $H$ is generated by $\Lambda$ and its identity component, $N$,

(1.1.3) $N$ is nilpotent.

A Riemannian manifold $(M, g)$ is called $(\rho, k)$-round at $p \in M$, if there exist $V, \widetilde{V}, H$ satisfying (1.1.1)-(1.1.3) and the following additional conditions:

(1.1.4) $V$ contains the metric ball, $B_{p}(\rho)$, of radius $\rho$ centered at $p$.

(1.1.5) The injectivity radius at all points of $\widetilde{V}$ is $>\rho$.

(1.1.6) $\sharp(H / N)=\sharp(\Lambda / \Lambda \cap N) \leq k$.

A metric, $g$, is called $(\rho, k)$-round if it is $(\rho, k)$-round at $p$, for all $p$.

Modulo the choice of $(\rho, k), V$ has a normal covering space with bounded geometry and a covering group that is almost nilpotent. By (1.1.5), if the injectivity radius at $p$ is $<\rho / k$, then the metric, $g$, has nontrivial local symmetries near $p$; i.e., the orbit, $H(\tilde{p})$, of $\tilde{p} \in \pi^{-1}(p)$, under $H$, has positive dimension.

If $(M, g)$ is $(\rho, k)$-round, it follows that the projected orbit, $\pi(H(\tilde{p}))$, contains those sufficiently collapsed directions corresponding to short geodesic loops that are homotopically nontrivial in $V$. The $(\rho, k)$-round metrics constructed in this paper actually have a stronger property. Namely, the orbits 
(have small diameter and) actually contain all sufficiently collapsed directions (again modulo the choice of $k$ ). One way of formulating this more precisely is to say that the orbit space has $(\rho, k)$-bounded geometry in a suitable sense. (See Definition 8.4 for the concept of $(\rho, k)$-bounded geometry of the orbit space and Remark 8.10; see also Appendix 1.)

Example 1.2. Let $G$ be a connected Lie group and $\widetilde{g}$ be a left invariant metric. Then, for each discrete subgroup $\Lambda$ of $G$, the quotient metric, $g$, on the quotient space $M=\Lambda \backslash G$ is $(\rho, 1)$-round, where $\rho$ depends on $\tilde{g}$ but is independent of $\Lambda$. This is a restatement of Zassenhaus's theorem, which asserts that every discrete subgroup of $G$ generated by small elements is contained in a nilpotent subgroup of $G$. (See [GLP, 8.44].) More generally, put $\widetilde{M}=G / K$, for some compact subgroup $K$ of $G$. Let $\widetilde{g}$ be a $G$-invariant metric on $\widetilde{M}$. Take a discrete subgroup $\Lambda$ of $G$ acting freely on $\widetilde{M}$. Then, by Zassenhaus's theorem, we conclude that the quotient metric $g$ on $M=\Lambda \backslash \widetilde{M}$ is $(\rho, k)$ round, where $\rho, k$ are independent of $\Lambda$.

Let $\nabla^{g}$ denote the Levi Civita connection of $g$.

Our first main result is

Theorem 1.3 (Symmetrization). For all $\varepsilon>0$ and $n \in \mathbb{Z}_{+}$, there exists $\rho>0$ and $k \in \mathbb{Z}_{+}$such that if $\left(M^{n}, g\right)$ is a complete Riemannian manifold with $|K| \leq 1$, then there is a $(\rho, k)$-round metric, $g_{\varepsilon}$, with

$$
\begin{aligned}
& e^{-\varepsilon} g<g_{\varepsilon}<e^{\varepsilon} g \\
& \left|\nabla^{g}-\nabla^{g_{\varepsilon}}\right|<\varepsilon \\
& \left|\left(\nabla^{g_{\varepsilon}}\right)^{i} R_{g_{\varepsilon}}\right|<c(n, i, \varepsilon) .
\end{aligned}
$$

One might ask whether Theorem 1.3 can be strengthened to the assertion that in all instances there exists a $(\rho, k)$-round metric, $g_{\varepsilon}$, such that either $\rho \geq \rho(n, \varepsilon), k \leq k(n)$ or $\rho \geq \rho(n), k \leq k(n, \varepsilon)$. However, this turns out to be false; see Examples 8.11, 8.12.

Now let $M$ be a smooth manifold and let $\mathfrak{g}$ be a sheaf of connected Lie groups on $M$. Let $\underline{\mathfrak{g}}$ be the associated sheaf of Lie algebras.

Definition 1.4. An action of $\mathfrak{g}$ is a (Lie algebra) homomorphism, $h$, of $\mathfrak{g}$ into the sheaf of smooth vector fields on $M$.

A metric, $g$, is called invariant for $\mathfrak{g}$ if $h(\underline{\mathfrak{g}})$ is a sheaf of local Killing fields for $g$.

Note that if $\pi: \widetilde{M} \rightarrow M$ is a local homeomorphism, then there is an induced action, $\pi^{*}(h)$, of the pullback sheaf, $\pi^{*}(\mathfrak{g})$.

A curve $c:(a, b) \rightarrow M$ is called an integral curve if $c \subset V$ for some open set, $V$, and $c$ is everywhere tangent to the image, $h(X)$, of some section $X \in \mathfrak{g}(V)$ (i.e,. $\left.c^{\prime}(s)=h(X)(c(s))\right)$. A set $Z \subset M$ is called invariant if $c \subset Z$, for all such $c$ with $c \cap Z \neq \varnothing$. The unique minimal invariant set containing $p$ is called the orbit, $\mathscr{O}_{p}$, of $p$. Clearly, $M$ is the union of its orbits.

Let $h$ be an action of a sheaf, $\mathfrak{n}$, of simply connected nilpotent Lie groups. Let $g$ be a $(\rho, k)$-round metric and let $N_{0}, V$, etc., be as in (1.1.1)-(1.1.6). 
Definition 1.5. $(\mathfrak{n}, h)$ defines a nilpotent Killing structure, for $g$, if for all $p$, we can choose $H, V, \widetilde{V}$ as follows. There is an invariant neighborhood $U$ and normal covering, $\widetilde{U} \subset \widetilde{V}$, such that:

(1.5.1) $\pi^{*}(h)$ is the infinitesimal generator of a (necessarily unique) action of the group, $\pi^{*}(\mathfrak{n})(\widetilde{U})$, whose kernel, $K$, is discrete. $N_{0}=\pi^{*}(\mathfrak{n})(\widetilde{U}) / K$ and the action of $N_{0} \mid \widetilde{U}$ is the quotient action.

(1.5.2) For all $W \subset \widetilde{U}$ such that $W \cap \pi^{-1}(p) \neq \varnothing$, the structure homomorphism, $\pi^{*}(\mathfrak{n})(\widetilde{U}) \rightarrow \pi^{*}(\mathfrak{n})(W)$ is an isomorphism.

(1.5.3) The neighborhood $U$ and covering $\widetilde{U}$ can be chosen independent of $p$, for all $p \in \mathscr{O}_{p}$.

Clearly, the metric $g$ in Definition 1.5 is an invariant metric for $(\mathfrak{n}, h)$.

A structure is called pure if the dimension of the stalk is locally constant.

Before going to the next example, we will recall some elementary (but confusing) facts.

Let $H$ be a Lie group. The diffeomorphisms of $H$ obtained by integrating right invariant vector fields are left translations. Conversely, integrating left invariant vector fields yields right translations.

In particular, given a left invariant metric on $H$, the right invariant fields are Killing fields but left invariant fields need not be.

Example 1.6. Let $N$ be a simply connected Lie group and $\Lambda \subset N$ a discrete subgroup. The quotient sheaf, $\mathfrak{n}$, of the constant sheaf, $N \times N \rightarrow N$, by the action, $\lambda:\left(n_{0}, n\right) \rightarrow\left(\lambda n_{0} \lambda^{-1}, \lambda n\right)$ has an action on $\Lambda \backslash N$ induced by left multiplication on $N$. The image sheaf, $h(\mathfrak{n})$, is the sheaf of locally defined right invariant vector fields. Any left invariant metric on $N$ induces an invariant metric on $\Lambda \backslash N$ for the action of this sheaf. It follows that $(\mathfrak{n}, h)$ defines a nilpotent Killing structure.

Note, however, that the standard collapsing construction for $\Lambda \backslash N$ involves inhomogeneous scaling of the left invariant metric and hence of the lengths of the left invariant vector fields (see $[\mathrm{BK}]$ ). The right action of $N$ generates the left invariant fields and gives rise to the nilpotent collapsing structure in this case. As indicated above, typically, the right action of $N$ on $\Lambda \backslash N$ does not give rise to a nilpotent Killing structure, because there is no metric that it leaves invariant.

Let $M, g, g_{\varepsilon}$ be as in Theorem 1.3. Our second main result is

Theorem 1.7. The $(\rho, k)$-round metric, $g_{\varepsilon}$, can be chosen such that there is a nilpotent Killing structure, $\mathfrak{N}$, for $g_{\varepsilon}$ whose orbits are all compact with diameter $<\varepsilon$.

Remark 1.8. The structure described in Theorem 1.7 can be viewed as generalizing the system of fibrations with nilpotent fibre and locally symmetric base that is known to exist near infinity on a noncompact locally symmetric space of finite volume.

Remark 1.9. Theorem 1.7 also provides an alternative means of obtaining an $F$-structure of positive rank on the collapsed part of $M$. In fact, replacing each 
Lie algebra of local sections, $\mathfrak{n}(U)$, by its center leads to the existence of an $F$-structure.

Open Problem 1.10. Suppose that the original metric, $g$, in Theorems 1.3 and 1.7 is Kähler, Einstein, etc. Can one take $g_{\varepsilon}$ within the same category?

In spirit, our construction of the nilpotent Killing structure is similar to the construction of the $F$-structure in [CG4]. But in carrying out the details, we use the framework of [F1-F3].

As in [CG4], we will fit together a collection of locally defined pure structures. Initially, the collection is organized in such a way that on nonempty intersections of their domains, the structures fit approximately, one inside another. Then using a suitable stability property they are perturbed so as to fit together exactly.

In [CG4] the locally defined pure structures are constructed on the scale of the injectivity radius, with the help of a result on local approximation by complete flat manifolds; see [CG4, §3]. The stability property is a consequence of the stability of compact group actions (in particular of torus actions); see [CG4, $\S 1]$. Here, we work on length scales that, though small, may be arbitrarily large compared to the injectivity radius. We also work with nilpotent groups, which typically have no compact quotient groups. As a consequence, neither of the above-mentioned basic tools is available.

Following the approach of [F1-F3], we will construct an $O(n)$-equivariant nilpotent Killing structure on the frame bundle. This structure induces the desired nilpotent structure on the base. The requirement of maintaining $O(n)$ equivariance at all stages of the construction introduces some technical problems; see in particular $\S 3$. They are handled by averaging arguments, some of which are very similar to those used to prove the stability of compact group actions. In addition, we use Malcev's rigidity theorem for discrete cocompact subgroups of nilpotent groups, which serves as a partial replacement for the stability of torus actions.

The preliminaries on which our construction is based are given in $\S \S 2-4$. The construction is carried out in $\S \S 5-8$ and is organized as follows.

In $\S \S 5-6$ we manufacture an $O(n)$-equivariant collection of local fibrations of the frame bundle such that if a pair of fibres from two of these fibrations intersect, then one fibre contains (or is equal to) the other.

In $\S 7$ flat affine structures are introduced on the fibres. Then, the fibrations are readjusted so that for a pair of fibres as above, the smaller is totally geodesic in the larger, with respect to these affine structures. The affine structures give rise to a nilpotent Killing structure on the frame bundle. Those fibres that are not contained in any other are the orbits of this structure. Their images in the base are the orbits of the structures we are seeking.

In $\S 8$ we check that the nilpotent structure and metric on the frame bundle do indeed induce the desired objects on the base.

Before giving a more detailed summary of the contents of the paper, we explain the following basic point that was alluded to in the previous section.

A manifold is called $A$-regular if for some nonnegative sequence $A=\left\{A_{i}\right\}$, we have

$$
\left|\nabla^{i} R\right| \leq A_{i}
$$


By the following result of Abresch [A] (see also [Ba, BMR, Shi]), we will ultimately (in $\S 8$ ) be able to replace the given metric in Theorems 1.3 and 1.7 by one that is $A$-regular, where

$$
A_{i}=A_{i}(n, \varepsilon)
$$

and $\varepsilon$ is as in Theorems 1.3 and 1.7. Thus, prior to $\S 8$ we will always work with manifolds that are $A$-regular with $A_{0}=1$.

Theorem 1.12 (Abresch). On the set of complete Riemannian manifolds, $\left(M^{n}, g\right)$, with $|K| \leq 1$, there exists for all $\varepsilon>0$, a smoothing operator, $g \rightarrow S_{\varepsilon}(g)=\tilde{g}$, such that

(1.12.1) $e^{-\varepsilon} g \leq \tilde{g} \leq e^{\varepsilon} g$,

(1.12.2) $|\nabla-\widetilde{\nabla}| \leq \varepsilon$,

(1.12.3) $\left|\widetilde{\nabla}^{i} \widetilde{R}\right| \leq A_{i}(n, \varepsilon)$.

Moreover, at $p \in M^{n}$, the value of $\tilde{g}$ depends only on $g \mid B_{p}\left(\frac{1}{4}\right)$. Finally, any isometry of $g$ is also an isometry of $\tilde{g}$.

Note that since $\tilde{g}(p)$ depends only on $g \mid B_{p}\left(\frac{1}{4}\right)$, the completeness assumption can be removed, provided one stays away from $\bar{M} \backslash M$ (where the bar denotes metric space completion).

In $\S 2$ we give a new proof of the fibration theorem of [F1], in the local equivariant form that we need. The projection map of the fibration is obtained by regularizing a Hausdorff approximation. Although there is some freedom in choosing the scale on which the regularization is performed, for the application in $\S \S 5-8$, it is important that the scale is chosen to be that of the injectivity radius of the base space.

In $\S 3$ we remove the dependence on the base point in Ruh's theorem by averaging the base point dependent choices of the flat connection that occur in the initial step of the proof. Since this procedure and the remainder of Ruh's arguments are both canonical, we immediately obtain an equivariant and parameterized version of his theorem.

In $\S 4$ we observe that the affine structures on the fibres introduced in $\S 3$ allow us to define in a canonical way, a pure nilpotent Killing structure on the total space of the fibration (we also construct a canonical metric that is invariant for the structure). Clearly, on a given fibre we can speak of the local right invariant fields. But the issue is to define these fields (locally) on the total space itself. For this, we note that the affine equivalence that identifies neighboring fibres is unique up to elements of the identity component, $\operatorname{Aff}^{0}(\Lambda \backslash N)$, of $\operatorname{Aff}(\Lambda \backslash N)$. One sees easily that $\operatorname{Aff}^{0}(\Lambda \backslash N) \subset N_{R}$, the subgroup of $\operatorname{Aff}(N)$ consisting of right translations. Since $N_{R}$ acts trivially on right invariant fields, it follows that there is a canonical $1-1$ correspondence between local right invariant fields (at nearby points) of neighboring fibres.

In $\S 5$ (using the results of $\S 2$ ) we select a system of $O(n)$-equivariant, local fibrations of the frame bundle with almost flat fibres. On the intersections of their domains, these fit approximately, one inside another. To achieve this requires a suitable mechanism for picking out which directions are to be considered collapsed in cases which might otherwise appear to be ambiguous. Lacking 
such a mechanism, we could wind up with fibrations whose domains intersect, but whose fibres do not satisfy the above relation of approximate containment. Essentially the same point had to be dealt with in [CG4, see $\S 5-b]$. Here we employ what amounts to a standard device from stratification theory.

In $\S 6$, by employing an inductive argument that depends on the result of Appendix 2, the local fibrations are modified $O(n)$-equivariantly, so that they fit, one inside another, on the intersections of their domains.

In $\S 7$ we complete the construction of the nilpotent Killing structure and invariant metric on the frame bundle, using an inductive argument like that of $\S 6$. For the construction of the Killing structure, the main part of the induction step can be described as follows. Note that as a consequence of $\S 3$, each fibre of a local fibration in $\S 6$ is endowed with a flat affine structure, affinely diffeomorphic to some $\Lambda \backslash N$. Consider a pair of fibrations, $\mathscr{F} \subset{ }_{t} \subset \mathscr{F}_{s}$ as in $\S 6$ (i.e., the fibres of $\mathscr{F}_{t}$ are contained in those of $\mathscr{F}_{s}$ ). By $\S 4$, the fibres carry affine structures that determine a local left action. However, the inclusion $\mathscr{F}_{t} \subset \mathscr{F}_{s}$ need not be compatible with the affine structures. Using Malcev's rigidity theorem, we find a unique subfibration, $\mathscr{F}_{t}^{\prime} \subset \mathscr{F}_{s}$, whose fibres are totally geodesic for the affine structure on the fibres of $\mathscr{F}_{s}$, and such that for each fibre of $\mathscr{F}_{t}$, there is a small motion carrying it onto some fibre of $\mathscr{F}_{t}^{\prime}$. Then, as in $\S 6$, we find a small $O(n)$-equivariant diffeomorphism that matches $\mathscr{F}_{t}$ with $\mathscr{F}_{t}^{\prime}$ such that the affine structure on $\mathscr{F}_{t}$ is carried into that of $\mathscr{F}_{t}^{\prime}$.

The remaining sections, $\S 8$ and the Appendices, require no further description at this point.

With minor variations, we will employ the same notation as in [F3, see $\S 1]$ and [F4, see $\S 7]$. In particular, we use:

(1.13.1) $d(\cdot, \cdot)$ : the distance function.

(1.13.2) $\quad B_{p}(D)=\{x \in M \mid d(p, x) \leq D\}$.

(1.13.3) $T B_{p}(D)=\left\{v \in T_{p} M|| v \mid<D\right\}$.

(1.13.4) $\pi_{1}(M, p ; \varepsilon)=\left\{\gamma: T B_{p}(\varepsilon) \rightarrow T B_{p}(2 \varepsilon) \mid \exp _{p} \circ \gamma=\exp _{p}\right\}$ : the pseudofundamental group. $\pi_{1}(M, p ; \varepsilon)$ has a pseudogroup structure and it acts on $T B_{p}(\varepsilon)$ with $T B_{p}(\varepsilon) / \pi_{1}(M, p ; \varepsilon)=B_{p}(\varepsilon)$.

(1.13.5) $d_{H}(X, Y)$ : the Hausdorff distance between $X$ and $Y$. When $X$ and $Y$ have isometric $G$-action, the $G$ Hausdorff distance is also denoted by $d_{H}(\cdot, \cdot)$.

(1.13.6) $\tau(\varepsilon \mid a, b, \ldots)$ denotes a positive number depending on the numbers in the parentheses and satisfying $\lim _{\varepsilon \rightarrow 0} \tau(\varepsilon \mid a, b, c, \ldots)=0$, for each fixed $a, b, c, \ldots$.

(1.13.7) If $\left\{A_{i}\right\}$ is a positive sequence $c(\cdot, A, \cdot)$ will denote a generic constant depending on finitely many of the $A_{i}$ (and possibly on some other parameters).

\section{Preliminaries}

\section{SMOOTHING HAUSDORFF APPROXIMATIONS}

In this section we give a new proof of the fibration theorem of [F1, F3] (see Theorem 2.6). 
A map $h: X \rightarrow Y$ of metric spaces will be called an $\delta$-Hausdorff approximation if for all $x_{1}, x_{2}$

(2.1.1) $\left|d\left(x_{1}, x_{2}\right)-d\left(h\left(x_{1}\right), h\left(x_{2}\right)\right)\right| \leq \delta$;

(2.1.2) the range of $h$ is $\delta$-dense.

If $G$ is a group acting by isometries on $X$ and $Y$, then $h$ is called a $G$ - $\delta$ Hausdorff approximation if in addition, for all $g \in G, x \in X$,

(2.1.3) $d(h(g x), g h(x))<\delta$.

Let $\bar{V}$ denote the completion of the metric space $V$, and put

(2.2) $\partial V=\bar{V} \backslash V$,

(2.3) $V_{\eta}=\{v \in V \mid d(v, \partial V)>\eta\}$.

Now let $X^{n}, Y^{j}(j \leq n)$ be Riemannian manifolds such that for some sequence, $\left\{A_{i}\right\}$, with $A_{0}=1$,

(2.4.1) $X^{n}, Y^{j}$ are $\left\{A_{i}\right\}$-regular.

Assume in addition, that for all $y \in Y$ and some $l \leq 1$,

(2.4.2) inj $\operatorname{rad}_{y} \geq \min \left(l^{-1}, d(y, \partial Y)\right)$.

Let $G$ act on $X^{n}, Y^{j}$ by isometries. Let distances in $X_{\delta}^{n}, Y_{\delta}^{j}$ be measured in $X^{n}, Y^{j}$ respectively. If the $G$-Hausdorff distance, $d_{H}\left(X^{n}, Y^{j}\right)$, satisfies (2.4.3) $d_{H}\left(X^{n}, Y^{j}\right) \leq \delta / 10$,

with $\delta l^{-1} \leq \frac{1}{2}$, then there is a continuous $G$ - $\delta$-Hausdorff approximation, (2.4.4) $h: X_{\delta}^{n} \rightarrow Y^{j}$,

with $h\left(X_{\delta}^{n}\right) \supset Y_{3 \delta}^{j}$ (see [F4, GLP, GrK]). In what follows it will be convenient simply to assume the existence of a continuous, $G-\delta$-Hausdorff approximation, $h: X^{n} \rightarrow Y^{j}$.

A fibration, $f: X \rightarrow Y$, of Riemannian manifolds is called a $\theta$-almost Riemannian submersion if for all $y \in Y, x \in f^{-1}(y)$, and $V \in T X_{x}$, normal to $f^{-1}(y)$,

$$
e^{-\theta}\left|f_{*}(V)\right| \leq|V| \leq e^{\theta}\left|f_{*}(V)\right| .
$$

Let $B=\left\{B_{i}\right\}, i=1,2, \ldots$ A map, $f: X \rightarrow Y$, of Riemannian manifolds is called $B$-regular if

$$
\left|\nabla^{i} f\right| \leq B_{i} .
$$

Let $I I_{Z}$ denote the second fundamental form of $Z \subset X$.

Fix $\lambda \leq \lambda(n)$ sufficiently small.

Theorem 2.6. Let $X^{n}, Y^{j}$ satisfy (2.4.1), (2.4.2) and let $h: X^{n} \rightarrow Y^{j}$ be a continuous, G-equivariant, $\delta$-Hausdorff approximation, with $\left(\delta l^{-1}\right)^{1 / 2} \leq \lambda$. Then there exists G-equivariant $W^{n} \supset X_{l}^{n}$ and a fibration $f: W \rightarrow Y^{j}$ such that

(2.6.1) $\operatorname{dia}\left(f^{-1}(y)\right) \leq c(n, A) \delta$, for all $y \in f\left(W^{n}\right)$. In particular, $f^{-1}(y)$ is a connected manifold. 
(2.6.2) $f$ is a $c(n, A) \lambda$-almost Riemannian submersion.

(2.6.3) $\left|I I_{f^{-1}(y)}\right| \leq c(n, A) l^{-1}$, for all $y \in f\left(W^{n}\right)$.

(2.6.4) $f$ is $\left\{C_{i}(n, A)\left(1+\lambda^{2-n}\right) l^{1-i}\right\}$-regular.

(2.6.5) $f$ is G-equivariant.

(2.6.6) For $c^{2}(n, A) \delta l^{-1} \leq \xi(n)$, sufficiently small, $f^{-1}(y)$ is an almost flat manifold, for all $y \in f\left(W^{n}\right)$.

(2.6.7) $d(h, f) \leq c(n) \lambda l$.

The proof of Theorem 2.6 will occupy the remainder of this section and will require a number of lemmas.

Before going through the proof of Theorem 2.6, the reader may wish to glance at Example 2.29 below.

Proof of Theorem 2.6. Note that (2.6.6) is a direct consequence of (2.6.1), (2.6.3). Also, given the bound on $\nabla^{2} f$ and (2.6.2), the connectedness of $f^{-1}(y)$ can be proved by an argument like the one used to prove (A.2.3.2) of Appendix 2.

As for the remaining statements, we begin by noting that by an obvious scaling argument, we can assume $l=1$.

We will construct $f$ by regularizing the map $h$.

Given $x \in X^{n}$ and $\beta: X \rightarrow Y$, we define (2.7) $\tilde{\beta}:=\beta \exp _{x}$,

on the ball $T B_{x}(1)$, of radius 1 in the tangent space, $T_{x} X$.

Let $\zeta:[0,1] \rightarrow[0,1]$ be a smooth function such that $\zeta\left|\left[0, \frac{1}{3}\right] \equiv 1, \zeta\right|\left[\frac{2}{3}, 1\right]$ $\equiv 0,\left|\zeta^{\prime}\right| \leq 4,\left|\zeta^{\prime \prime}\right| \leq 12$. For $\tilde{x} \in T B_{x}(1)$, put

(2.8.1) $\chi_{\varepsilon}(\tilde{x})=\zeta\left(\varepsilon^{-1} d(0, \tilde{x})\right)$.

Then

(2.8.2) $\left|\nabla \chi_{\varepsilon}\right| \leq c(n) \varepsilon^{-1}$,

(2.8.3) $\left|\nabla^{2} \chi_{\varepsilon}\right| \leq c(n) \varepsilon^{-2}$.

Let $d \tilde{x}$ denote the volume form for the pullback metric on $T B_{x}(1)$. Consider (for fixed $x$ and small $\varepsilon$ ) the function,

(2.9.1) $y \rightarrow \int d^{2}(\tilde{\beta}(\tilde{x}), y) \chi_{\varepsilon}(\tilde{x}) d \tilde{x}$.

If $\beta\left(B_{x}(\varepsilon)\right) \subset B_{\beta(x)}(a)$, for some $a<\frac{\pi}{3}$, then the function in (2.9.1) is a weighted average of convex functions on the ball, $B_{\beta(x)}(3 a)$, and so, is itself convex on this ball. Clearly, it takes a unique minimum at some $y_{1} \in \overline{B_{\beta(x)}(2 a)}$. By definition, $y_{1}$ is the center of mass of $\tilde{\beta}$, weighted by the function $\chi_{\varepsilon}$.

Define the $\varepsilon$-regularization of $\beta$, by

(2.9.2) $\beta_{\varepsilon}(x):=y_{1}$.

We note that the $\varepsilon$-regularization can also be defined for a continuous function, $\xi$, on $T B_{x}(1)$, satisfying $\left(T B_{x}(\varepsilon)\right) \subset B_{\xi(0)}(a)$, by using the pullback metric. We continue to denote the $\varepsilon$-regularization of such a function by $\xi_{\varepsilon}$. In particular, for those functions, $\tilde{\beta}$, as in (2.7), that are pullbacks we see by in- 
spection that the following crucial relation holds (compare [CG3, Lemma 5.3]). On $T B_{x}(1)$,

$$
\text { (2.9.3) }(\tilde{\beta})_{\varepsilon}=\tilde{\beta}_{\varepsilon} \text {. }
$$

Now define (2.10) $f:=h_{\lambda}$.

Since $h$ is $G$-equivariant, it follows that $f$ satisfies (2.6.5) and by a standard argument, (2.6.4), (2.6.7) hold as well. However, (2.6.1)-(2.6.3) are not yet apparent.

In order to prove (2.6.1)-(2.6.3), we will compare $\tilde{h}$, with an auxiliary function, $k_{x}$, on $T B_{x}(2 \lambda)$. (From now on we just write $k$ for $k_{x}$.) The function $k$ has regularity properties like those that we are trying to establish for $f$. However, $k$ can be constructed directly since it is locally defined, allowed to depend on $x$, and not required to be the pullback of a function on $B_{x}(2 \lambda)$.

The crucial point will be to show that $k$ and $\tilde{h}$ are $c(n) \lambda^{2}$-close (see (2.11.1)). This degree of closeness will imply that the regularity properties of $\tilde{h}_{\lambda}=\tilde{f}$ (by (2.9.3)) are like those of $k_{\lambda}$. These, in turn, are like those of $k$, since $k$ is already regular. Finally (of course) the regularity properties of $f$ and $\tilde{f}$ are the same.

A priori, it is only clear that $k$ and $\tilde{h}$ are $c(n) \lambda$-close. This does not suffice for our purposes since it leads only to a bound on $\nabla f$ and not to the assertion that $f$ is an almost Riemannian submersion. It is in establishing the required closeness of $k$ and $\tilde{h}$ that the geometry of our setup enters (in essentially the same way as in [F1, §3]); see Lemmas 2.16 and 2.19.

In the lemmas that follow, $\widetilde{\nabla}$ denotes the Levi Civita connection of the pullback metric on $T B_{x}(1)$.

Lemma 2.11. For all $x \in X^{n}$, there is a function, $k: T B_{x}(2 \lambda) \rightarrow Y^{j}$, such that

(2.11.1) $d(k, \tilde{h}) \leq c(n, A) \lambda^{2}$,

(2.11.2) $k$ is a $c(n) \delta^{1 / 2}$-almost Riemannian submersion,

(2.11.3) $\left|\tilde{\nabla}^{2} k\right| \leq c(n, A)$.

\section{Lemma 2.12.}

(2.12.1) $k_{\lambda}$ is a $c(n) \lambda$-almost Riemannian submersion.

(2.12.2) $\left|\widetilde{\nabla}^{2} k_{\lambda}\right| \leq c(n, A)$.

\section{Lemma 2.13.}

(2.13.1) $\left|\widetilde{\nabla} \tilde{f}-\widetilde{\nabla} k_{\lambda}\right| \leq c(n, A) \lambda$.

(2.13.2) $\left|\widetilde{\nabla}^{2} \tilde{f}-\widetilde{\nabla}^{2} k_{\lambda}\right| \leq c(n, A)$

Essentially, to get Lemma 2.13, we can estimate the $i$ th derivative of the regularization of $(\tilde{h}-k)$ by $\lambda^{-i}$ times the quantity in (2.11.1) (see (2.8)). Similarly, the properties $(2.12 .1),(2.12 .2)$ are consequences of the corresponding properties (2.11.2), (2.11.3).

Indeed, Lemmas 2.12 and 2.13 would be standard in the familiar case $X^{n}=$ $R^{n}, Y^{j}=R^{j}$. In the present context, their proofs are straightforward, if slightly 
tedious, exercises in advanced calculus. Hence, the proofs of these lemmas will be omitted.

The proof of Lemma 2.11 will be given at the end of this section.

By combining Lemmas 2.12 and 2.13 we see that

(2.14) $f$ is a $c(n, A) \lambda$-almost Riemannian submersion if (2.6.2) and (2.6.3) holds.

Clearly, we can choose $W^{n} \supset X_{1}^{n}$ such that $f^{-1}(y) \subset W$ is compact for all $y \in f\left(W^{n}\right)$.

We now prove (2.6.1).

Note that by (2.6.3), if some fibre, $f^{-1}\left(y_{0}\right)$, has diameter, $\mu \delta$, then for all $y \in B_{y_{0}}\left(\frac{1}{2}\right)$,

(2.15.1) dia $\left(f^{-1}(y)\right) \geq c^{-1}(n, A) \mu \delta$.

In view of (2.14), it follows that for $\delta<c^{-1}(n)$, at least

(2.15.2) $c^{-1}(n, A) \mu \delta^{-j}$

balls of radius $\delta$ are required to cover $f^{-1}\left(B_{y_{0}}\left(\frac{1}{2}\right)\right)$.

But from the existence of the $\delta$-Hausdorff approximation $h$, it follows that at most

(2.15.3) $c(n) \delta^{-j}$

such balls are required. Therefore we get

(2.15.4) $c(n) c(n, A) \geq \mu$,

which gives (2.6.1).

In order to prove Lemma 2.11 we will need two auxiliary lemmas (compare $[\mathrm{F} 1, \mathrm{~F} 3])$.

Lemma 2.16. Let $X, Y$ be as in Theorem 2.6 and let $\sigma$ be a geodesic loop of length $l$ on $p \in X_{1}$. Let $\gamma$ be a minimal geodesic segment with $\frac{1}{3} l \leq L[\gamma] \leq l$ and $\gamma(0)=p$. Then

$$
\left|\chi\left(\gamma^{\prime}(0), \sigma^{\prime}(0)\right)-\frac{\pi}{2}\right| \leq c \max \left(l_{l}^{-1},\left(\delta l^{-1}\right)^{1 / 2}\right) .
$$

Proof. By scaling, it suffices to consider the case $l=1$.

The inequality

$$
\Varangle\left(\gamma^{\prime}(0), \sigma^{\prime}(0)\right) \geq \frac{\pi}{2}-c l
$$

is a direct consequence of Toponogov's theorem applied to the degenerate isoceles triangle with sides $\gamma, \gamma, \sigma$.

On the other hand, put

(2.18.1) $h\left(\gamma\left(\frac{1}{3}\right)\right)=\exp _{h(p)}(u)$,

and choose $p^{\prime}$ such that

(2.18.2) $d\left(h\left(p^{\prime}\right), \exp _{h(p)}(-u)\right) \leq \delta$.

By Toponogov's theorem, if $\zeta$ is minimal from $p$ to $p^{\prime}$, then (2.18.3) $\left|\not\left(\zeta^{\prime}(0), \gamma^{\prime}(0)\right)-\pi\right| \leq c \delta^{1 / 2}$. 
By using (2.17) with $\zeta$ in place of $\gamma$, we get (2.16.1) (compare [F1, §3]).

Lemma 2.19. Let $X^{n}, Y^{j}$ be as in Theorem 2.6. Let $\gamma_{1}, \gamma_{2}$ be minimal geodesics in $X^{n}$ joining the point $z$ to points $q_{1}, q_{2} \in X_{l}^{n}$ respectively. Assume that $\frac{1}{3} l \leq L\left[\gamma_{1}\right] \leq \frac{5}{12} l$ and that $d\left(q_{1}, q_{2}\right)=l<<\frac{1}{3} l$. Then

$$
\Varangle\left(\gamma_{1}^{\prime}(0), \gamma_{2}^{\prime}(0)\right) \leq c \max \left(l l^{-1},\left(\delta l^{-1}\right)^{1 / 2}\right) .
$$

Proof. By scaling it suffices to consider the case $l=1$.

Write $h\left(q_{1}\right)=\exp _{h(z)} v$ and put $y=\exp _{h(z)}(-v)$. Using the facts that $h$ is a $\delta$-Hausdorff approximation and that $Y^{j}$ has bounded geometry, we find by a standard comparison argument that

(2.20.1) $d\left(h\left(q_{2}\right), h(z)\right)+d(h(z), y)-d\left(h\left(q_{2}\right), y\right) \leq c_{1}(l+\delta)^{2}$.

(This quantity is the excess of the triangle with vertices $h\left(q_{2}\right), h(z), y$.) Let $w \in X$ be such that $d(h(w), y) \leq \delta$. Then we have

(2.20.2) $d\left(q_{2}, z\right)+d(z, w)-d\left(q_{2}, w\right) \leq c_{2}\left[(l+\delta)^{2}+\delta\right]$.

Thus, if $\tau$ is minimal from $z$ to $w$, Toponogov's theorem implies

(2.20.3) $\Varangle\left(\gamma_{2}^{\prime}(0), \tau^{\prime}(0)\right) \geq \pi-c_{3} \max \left(l, \delta^{1 / 2}\right)$.

Similarly,

(2.20.4) $d\left(q_{1}, z\right)+d(z, w)-d\left(q_{1}, w\right) \leq 3 \delta$,

and by Toponogov's theorem,

(2.20.5) $\Varangle\left(\gamma_{1}^{\prime}(0), \tau^{\prime}(0)\right) \geq \pi-c \delta^{1 / 2}$.

Our claim follows from (2.20.3) and (2.20.5).

Remark 2.21. In the proof of Lemma 2.16 we used only the lower bound on $K_{X}$; compare [Y]. But in Lemma 2.19 we also use the two-sided bound on $K_{Y}$. Proof of Lemma 2.11. Let $e_{1}, \ldots, e_{j}$ be an orthonormal frame at $h(x)$. Pick $x_{1} \cdots x_{j} \in X^{n}$ such that

$$
d\left(h\left(x_{i}\right), \exp _{h(x)}\left(\frac{1}{3} e_{i}\right)\right) \leq \delta .
$$

Let $x_{i}^{\prime} \in T X_{x}^{n}$, with $\exp _{x} x_{i}^{\prime}=x_{i}$ and $t \rightarrow \exp _{x} t x_{i}^{\prime}, t \in[0,1]$, a minimal segment from $x$ to $x_{i}$. For $p \in B_{x}(2 \lambda), p^{\prime} \in T B_{x}(2 \lambda) \subset T X_{x}$, put

(2.23.1) $\rho_{x_{i}}(p):=d\left(p, x_{i}\right)$,

(2.23.2) $\rho_{x_{i}^{\prime}}\left(p^{\prime}\right):=d\left(p^{\prime}, x_{i}^{\prime}\right)$.

We claim that Lemmas 2.16 and 2.19 imply

$$
\left|\tilde{\rho}_{x_{i}}-\rho_{x_{i}^{\prime}}\right| \leq c \lambda^{2} \text {. }
$$

For the moment, let us grant this. Then if we define $k$ by

$$
d\left(\exp _{h(x)}\left(\frac{1}{3} e_{i}\right), k\left(p^{\prime}\right)\right)=\rho_{x_{i}^{\prime}}\left(p^{\prime}\right), \quad i=1, \ldots, j,
$$

we get (2.11.1). Moreover, (2.11.2), (2.11.3) are direct consequences of the definition of $k$. 
To verify (2.24), fix $x_{i}$ as above and let $\gamma_{1}$ be minimal from $x_{i}$ to $x$. Take $p^{\prime} \in T B_{x}(2 \lambda) \subset T X_{x}$ and put $p=\exp _{x} p^{\prime}$. Let $\gamma_{2}$ be minimal from $x_{i}$ to $p$. Let $\tilde{\gamma}_{1}$ be the lift of $\gamma_{1}$ running from $x_{i}^{\prime}$ to $0 \subset T X_{x}$. Finally, let $\tilde{\gamma}_{2}$ be the lift of $\gamma_{2}$ with initial point $x_{i}^{\prime}$.

Since by Lemma 2.19,

(2.26.1) $\Varangle\left(\gamma_{1}^{\prime}(0), \gamma_{2}^{\prime}(0)\right) \leq c \lambda$,

it follows that the end point, $p^{\prime \prime}$, of $\tilde{\gamma}_{2}$ lies in $T B_{x}(c \lambda)$. Let $\tilde{\sigma}$ be minimal from $p^{\prime \prime}$ to $p^{\prime}$. Then we have

(2.26.2) $L[\tilde{\sigma}] \leq c \lambda$.

The projection of $\tilde{\sigma}$ is a geodesic loop, $\sigma$, on $p$, of length $L[\sigma]=L[\tilde{\sigma}]$. It follows from Lemma 2.16 that

$$
\begin{aligned}
\Varangle\left(\sigma^{\prime}(0),-\gamma_{2}^{\prime}(l)\right) & =\Varangle\left(\tilde{\sigma}^{\prime}(0),-\tilde{\gamma}_{2}^{\prime}(l)\right) \\
& \leq \frac{\pi}{2}+c \lambda .
\end{aligned}
$$

Using (2.26.2), (2.27), and a standard comparison argument, we get

$$
\begin{aligned}
d\left(p^{\prime}, x_{i}^{\prime}\right) & \leq d\left(p^{\prime \prime}, x_{i}^{\prime}\right)+(c \lambda)(c \lambda), \\
& =d\left(p^{\prime \prime}, x_{i}^{\prime}\right)+c^{2} \lambda^{2} .
\end{aligned}
$$

Since

(2.28.2) $d\left(p^{\prime}, x_{j}^{\prime}\right)=\rho_{x_{j}^{\prime}}\left(p^{\prime}\right)$,

(2.28.3) $d\left(p^{\prime \prime}, x_{j}^{\prime}\right)=\tilde{\rho}_{x_{j}}\left(p^{\prime}\right)$,

this suffices to complete the proof.

Example 2.29. For $a>0$, consider the annulus, $a<r<2$, in $R^{2}$. Let $X_{a, \delta}^{2}$ (where $\delta=2 \pi / N)$ denote its quotient by the action of $\mathbb{Z}_{N} ;(r, \theta) \rightarrow(r, \theta+$ $2 \pi / N)$. Let $Y^{\prime}$ be the open interval, $(a, 2)$. Then the map $h((r, \theta)):=r$ is a Riemannian submersion and a $\delta$-Hausdorff approximation. But no matter how small we take $\delta$, the second fundamental form of the fibres has norm $1 / r$, which blows up as $a \rightarrow 0$. Clearly, no smoothing procedure will improve this situation. This confirms the necessity of restricting $f$ in Theorem 2.6 to points that are far from $\partial X^{n}$ (e.g., to $X_{1}^{n}$ ) independent of how small we take $\lambda$.

The reader may also wish to verify Lemma 2.11 directly in the context of this example.

For the application in $\S 5$, we will need the following sharpening of (2.6.2).

Let $f$ be as in Theorem 2.6 and let $v$ be a tangent vector at $x \in f^{-1}(y)$, with $v$ orthogonal to $f^{-1}(y)$.

Proposition 2.30. There exists a geodesic $\gamma$, with $\gamma(0)=x, \gamma \mid\left[0, \frac{1}{3} l\right]$ minimal, and

$$
\Varangle\left(\gamma^{\prime}(0), v\right) \leq c(n, A)\left(\delta l^{-1}\right)^{1 / 2} .
$$

Let $\tau$ be the minimal geodesic from $f(\gamma(0))$ to $f\left(\gamma\left(\frac{1}{3} l\right)\right)$. Then

$$
\Varangle\left(d f(v), \tau^{\prime}(0)\right) \leq c(n, A)\left(\delta l^{-1}\right)^{1 / 2} .
$$


Proof. By Lemma 2.13, it suffices to verify the corresponding assertions for the map $k$, for which they are clear by inspection.

\section{EQUIVARIANT AND PARAMETRIZED VERSION OF THE THEOREM ON ALMOST FLAT MANIFOLDS}

The main result of this section is concerned with fibrations such as those obtained in Theorem 2.6. To prove it, we show that one can canonically remove the dependence on the base point in the initial step of the proof of Ruh's theorem [R] (see also [Gh1]). Thus, initially we will be concerned with a single almost flat manifold.

Let $N$ be a nilpotent Lie group (which need not be simply connected). The canonical connection, $\nabla^{\text {can }}$, on the tangent bundle of $N$, is, by definition, the unique connection that makes all the left invariant vector fields parallel. Let $N_{L} \propto$ Aut $N$ be the skew product of $N_{L}$ and Aut $N\left(N_{L}\right.$ denotes an isomorphic copy of $N$ acting on $N$ by left multiplication). It is easy to see that this group coincides with the group, $\operatorname{Aff}\left(N, \nabla^{\text {can }}\right)$, the group of all affine transformations of $\left(N, \nabla^{\mathrm{can}}\right)$. If $\Lambda \subset \operatorname{Aff}\left(N, \nabla^{\text {can }}\right)$ is a subgroup whose action on $N$ is properly discontinuous, we can define the induced connection, $\nabla^{\text {can }}$, on the quotient space $\Lambda \backslash N$.

Remark 3.1. The subgroups, $N_{L}$ and $N_{R}$, can be defined intrinsically, just using the affine structure of $\left(N, \nabla^{\text {can }}\right)$. The group $N_{L}$ is the kernel of the holonomy homomorphism, i.e., the subgroup that acts trivially on all globally parallel fields. The group $N_{R}$ is obtained by integrating these fields. On the other hand, the subgroup, Aut $N$, can be described as the isotropy group of the identity element, $e \in N$. Equivalently, it depends on a choice of base point in the affine homogeneous space $\left(N, \nabla^{\text {can }}\right)$. Thus, the specific isomorphism, $\operatorname{Aff}\left(N, \nabla^{\text {can }}\right) \simeq N_{L} \propto \operatorname{Aut}(N)$ depends on a choice of base points as well.

Let $Z^{m}$ be an $A$-regular Riemannian manifold with $A_{0}=1$ and diameter, $\delta \leq \xi(m)$. In [R] Ruh observed that the results of [G] allow one to associate to each point, $z \in Z^{m}$, a flat orthogonal connection, $\nabla^{z}$, such that:

(3.2.1) For $p, q \in Z^{m}$, there is a gauge transformation, $g^{p, q}$, carrying $\nabla^{q}$ to $\nabla^{p}$.

(3.2.2) $g^{p, q}$ can be chosen such that $\mid g^{p, q}-$ Ident $|\leq c(m) \delta,| \nabla^{i} g^{p, q} \mid \leq$ $c(A, i) \delta$, where Ident denotes the identity element of the gauge group.

(3.2.3) For all $z,\left|\nabla^{i}\left(\nabla^{\mathrm{LC}}-\nabla^{z}\right)\right| \leq c(m, A, i) \delta$, where $\nabla^{\mathrm{LC}}$ denotes the Levi Civita connection of the underlying metric.

(3.2.4) The connection, $\nabla^{z}$, depends smoothly on $z$, (with estimates like those above on derivatives with respect to $z$ ).

(3.2.5) The holonomy group of $\nabla^{z}$ has order $<\omega_{m}$ (see, however, Remark 3.9).

Ruh went on to show that for some simply connected nilpotent Lie group, $N$, and discrete subgroup, $\Lambda \subset \operatorname{Aff}\left(N, \nabla^{\text {can }}\right)$, with $\#\left(\Lambda \cap N_{L} \backslash \Lambda\right)$ equal to the order of the holonomy group, one can associate to each connection, $\nabla^{z}$, a gauge transformation conjugating $\nabla^{z}$ into a connection isomorphic to the connection $\nabla^{\text {can }}$ on $\Lambda \backslash N$. The fact that $\Lambda \backslash N$ is actually the same for all $z$, follows from 
Malcev's rigidity theorem, (see [Rag, BK] and Theorem 3.7).

We now show that by suitably averaging the family of connections, $\nabla^{z}$, we can obtain a canonical gauge equivalent flat connection associated to the Riemannian structure (and not depending on a choice of base point). The connection isomorphic to $\nabla^{\text {can }}$, associated to this one by Ruh's construction, depends smoothly on the underlying metric and is automatically invariant under all of its isometries. From this, the main result of this section follows immediately.

We now explain the averaging procedure. Let $\mathscr{P}$ denote the bundle associated to the frame bundle, $F Z^{m}$, via the adjoint representation. Each fibre of $\mathscr{P}$ has a natural group structure isomorphic to $O(m)$. The gauge group is the space of sections of $\mathscr{P}$, equipped with the group structure induced by pointwise multiplication. It has a natural action on $F Z^{m}$, which commutes with the action of $O(m)$. Hence it also acts on the space of connections.

A connection, $\nabla$, on $F Z^{m}$ induces a connection on $\mathscr{P}$. The group, $K(\nabla)$, of gauge transformations fixing $\nabla$ is easily seen to be the group of parallel sections of $\mathscr{P}$ with respect to the induced connection. Let $\mathscr{K}(\nabla) \subset \mathscr{P}$ be the bundle whose fibre at $z \in Z^{m}$ is gotten by evaluating at $z$, the sections of $K^{0}(\nabla)$, the identity component of $K(\nabla)$. Then $\mathscr{K}(\nabla)$ is canonically trivial.

Let $g^{p, q}$ be as in (3.2.1). Put

$$
\begin{aligned}
g^{p, q}(z) & =h^{p, q}(z) k^{p, q}(z) \\
& =e^{U(z)} e^{V(z)}
\end{aligned}
$$

where $V(z)$ is in the Lie algebra of $\mathscr{K}(\nabla)_{z}$ and for all $z$,

(3.3.2) $\langle U(z), V(z)\rangle=0$.

The inner product in (3.3.2) comes from the negative of the Killing form. Since $g^{p, q}$ is close to the identity, it is uniquely defined up to right multiplication by an element of $K^{0}\left(\nabla^{q}\right)$. It follows that $h^{p, q}$ is independent of the particular choice of $g^{p, q}$. Also, there is a unique choice of $g^{p, q}$ that satisfies

(3.3.3) $\int_{Z^{m}} V(z) d z=0$,

where $d z$ is the normalized Riemannian volume element, for which

(3.3.4) $\int_{Z^{m}} d z=1$.

This is an immediate consequence of center of mass construction for the compact Lie group $K^{0}(\nabla)$; see [BK, $\S 8$ ].

Suppose $g^{p, q}, g^{q, w}, g^{p, w}$ are normalized as in (3.3.2), (3.3.3). Put

$$
g^{p, q}=e^{U_{1}} e^{V_{1}}, \quad g^{q, w}=e^{U_{2}} e^{V_{2}}, \quad g^{p, w}=e^{U_{3}} e^{V_{3}}
$$

where $V_{1} \in \mathscr{K}\left(\nabla^{q}\right), V_{2}, V_{3} \in \mathscr{K}\left(\nabla^{w}\right)$. Assume

(3.4.2) $\left\|U_{j}\right\|,\left\|V_{j}\right\|<\eta<<1$.

Then since the product of elements in a Lie group that are close to the identity is commutative modulo higher order terms,

$$
\begin{aligned}
g^{p, q} g^{q, w} & =e^{U_{1}} g^{q, w}\left(g^{q, w}\right)^{-1} e^{V_{1}} g^{q, w} \\
& =e^{U_{1}+U_{2}} e^{V_{2}+V_{1}^{\prime}}+O\left(\eta^{2}\right)
\end{aligned}
$$

Here,

(3.4.4) $\left(g^{q, w}\right)^{-1} e^{V_{1}} g^{q, w}:=e^{V_{1}^{\prime}}$. 
Thus, $V_{1}^{\prime} \in \mathscr{K}\left(\nabla^{w}\right)$. It follows that

(3.4.6) $\int_{Z^{m}}\left(V_{2}+V_{1}^{\prime}\right) d z=0$,

which easily implies that

(3.4.7) $g^{p, q} g^{q, w}=g^{p, w}+O\left(\eta^{2}\right)$.

Set

For fixed $w$ and variable $p$ in (3.4.1), we write $U_{3}=U_{3}(p), V_{3}=V_{3}(p)$.

$$
\text { (3.5.1) } g^{w}=e^{\int_{Z} U_{3}(p) d p} \cdot e^{\int_{Z} V_{3}(p) d p} \text {. }
$$

By using (3.4.7), we obtain

(3.5.2) $g^{q} g^{q, w}=g^{w}+O\left(\eta^{2}\right)$.

Hence, if we put

(3.5.3) $\nabla_{1}^{w}=g^{w}\left(\nabla^{w}\right)$,

we get

(3.5.4) $\nabla_{1}^{q}=\nabla_{1}^{w}+O\left(\eta^{2}\right)$.

By iterating the above construction, we obtain convergent sequences, $\nabla_{1}^{q}$, $\nabla_{2}^{q}, \ldots$ such that for all $q, w$

(3.5.6) $\nabla^{\infty}:=\lim _{j \rightarrow \infty} \nabla_{j}^{q}=\lim _{j \rightarrow \infty} \nabla_{j}^{w}$

is independent of the base point and, in particular, invariant under the isometry group of $Z^{m}$.

We now turn to our main result, Proposition 3.6.

Let $X^{n}, Y^{j}$ be $A$-regular Riemannian manifolds, with $A_{0}=1$, and let $f: X^{n} \rightarrow Y^{j}$ be a $\left\{C_{i} l^{1-i}\right\}$-regular, 1-almost Riemannian submersion, where $C_{i}=C_{i}(n, A)$. (This normalization corresponds to that of Theorem 2.6 but no assumption on inj $\operatorname{rad}_{y}, y \in Y^{j}$, is required here.) Assume that $G$ acts on $X^{n}, Y^{j}$ by isometries and that $f$ is $G$-equivariant.

Let $\nabla^{y, \text { LC }}$ denote the Levi Civita connection for the induced metric on $f^{-1}(y)$. Suppose that $\operatorname{dia}\left(f^{-1}(y)\right) \leq \delta$ and $\left|I I_{f^{-1}(y)}\right| \leq c l^{-1}$, with $c \delta l^{-1} \leq$ $\xi(n)$, where $c=c(n, A)$ and $\xi(n)$ is so small that $f^{-1}(y)$ is almost flat. Let $\nabla^{y, *}$ denote the affine flat connection on $f^{-1}(y)$ obtained by applying the construction of $[\mathrm{R}]$ (or [Gh1]) to the connection, $\nabla^{y, \infty}$, associated to $\nabla^{y, \mathrm{LC}}$ via (3.5.6). Thus, $\left(f^{-1}(y), \nabla^{y, *}\right)$ is affinely diffeomorphic to some $\left(\Lambda \backslash N, \nabla^{\text {can }}\right)$ with \# $\left(\Lambda \cap N_{L} \backslash \Lambda\right) \leq \omega_{n}$ (see, however, Remark 3.9).

Let $y$ vary and regard, $\nabla^{y, \mathrm{LC}}-\nabla^{y, \infty}, \nabla^{y, \mathrm{LC}}-\nabla^{y, *}$ as tensor fields on $X^{n}$, by putting $\nabla_{V}^{y, \text { LC }}-\nabla_{V}^{y, \infty}=0, \nabla_{V}^{y, \text { LC }}-\nabla_{V}^{y, *}=0$, for $V$ orthogonal to $f^{-1}(y)$.

\section{Proposition 3.6.}

(3.6.1) $\left|\nabla^{i}\left(\nabla^{y, \mathrm{LC}}-\nabla^{y, \infty}\right)\right| \leq C_{i}(n, A) \delta l^{-(2+i)}$.

(3.6.2) $\left|\nabla^{i}\left(\nabla^{y, \infty}-\nabla^{y, *}\right)\right| \leq C_{i}(n, A) \delta l^{-(2+i)}$. 
(3.6.3) If $h \in G$, then $h:\left(f^{-1}(y), \nabla^{y, *}\right) \rightarrow\left(h\left(f^{-1}(y)\right), \nabla^{h(y), *}\right)$ is an affine diffeomorphism.

Proof. By our previous discussion, Ruh's method yields a family of affine flat structures on the fibres and it is straightforward to check that the conditions of the proposition hold. Each $Z_{y}$ is affine equivalent to some $\left(\Lambda_{y} \backslash N_{y}, \nabla^{\text {can }}\right)$. Moreover, since affine structures of this type cannot occur in nontrivial families, $\Lambda_{y} \backslash N_{y}=\Lambda \backslash N$ is actually independent of $y$. This is a weak generalization of the second Bieberbach theorem (the uniqueness of the affine structure on a compact flat Riemannian manifold; see [Char]). For completeness, we give the argument (see, however, Remark 3.9).

A local trivialization of our fibration over an open neighborhood, $U$, of $y \in Y$, induces isomorphisms, $\Lambda_{y^{\prime}} \simeq \pi_{1}\left(Z_{y^{\prime}}\right) \simeq \pi_{1}\left(Z_{y}\right) \simeq \Lambda_{y}$. The holonomy homomorphisms vary continuously and, by (3.6.1), have finite image. It follows that the identifications, $\Lambda_{y^{\prime}} \simeq \Lambda_{y}$, respects the kernels, $\Lambda_{y^{\prime}} \cap N_{y^{\prime}}, \Lambda_{y} \cap N_{y}$ of the holonomy homomorphisms. These are cocompact subgroups of the groups $N_{y^{\prime}}, N_{y}$. By a theorem of Malcev the isomorphisms $\Lambda_{y^{\prime}} \cap N_{y^{\prime}} \simeq \Lambda_{y} \cap N_{y}$ extend uniquely to isomorphisms, $N_{y^{\prime}} \simeq N_{y}$.

Theorem 3.7 (Malcev). Let $N_{1}, N_{2}$ be simply connected nilpotent Lie groups and $\Lambda \subset N_{1}$ a cocompact subgroup. Then a homomorphism from $\Lambda_{1}$ to $N_{2}$ extends uniquely to $N_{1}$.

Now the following consequence of the affine center of mass construction for Lie groups [BK, $\S 8]$ implies the asserted rigidity of $\left(\Lambda_{y} \backslash N_{y}, \nabla^{\mathrm{can}}\right)$.

Proposition 3.8. Let $h_{t}: G_{1} \rightarrow G_{2}$ be a continuous family of homomorphisms of Lie groups such that for some subgroup $H \subset G_{1}$, of finite index, $h_{t} \mid H$ is independent of $t$. Then there is a continuous map, $t \rightarrow k_{t} \in G_{2}$ such that $h_{t}=k_{t} h_{0} k_{t}^{-1}$.

Proof. It suffices to consider $t$ so small that the affine center of mass, $k_{t}$, of the finite set $\left\{h_{t}^{-1} h_{0}(g) \mid g \in G_{1}\right\}$ is defined. As in Proposition 8.1.7 of [BK], this choice of $k_{t}$ has the required property.

Remark 3.9. The results of this section and the next will be applied in $\S 7$ to the local fibrations of the frame bundle constructed in $\S \S 5,6$. In that case, one actually has $\Lambda \subset N_{L}$; equivalently, the connection $\nabla^{\text {can }}$ on $\Lambda \backslash N$ is globally flat (see $\S 7$ and Appendix 1). Thus, Proposition 3.8 and the argument given in the proof of Proposition 3.6 are not needed for the construction of the nilpotent Killing structure.

\section{NilPOTENT KiLling STRUCTURES ON FIBRATIONS}

Let $Z \rightarrow X \stackrel{f}{\rightarrow} Y$ be a fibration acted on isometrically by a compact group $G$, such that the assumptions of Proposition 3.6 hold. By Proposition 3.6, each fibre carries a flat affine structure isomorphic to some $\Lambda \backslash N$.

Let $N_{\mathrm{Aff}(N)} \Lambda$ and $C_{\mathrm{Aff}(N)} \Lambda$ denote respectively the normalizer and centralizer of $\Lambda$ in $\operatorname{Aff}(N)$. Then 


$$
\operatorname{Aff}(\Lambda \backslash N) \simeq\left(N_{\mathrm{Aff}(N)} \Lambda\right) / \Lambda
$$

and since $\Lambda$ is discrete,

$$
\operatorname{Aff}^{0}(\Lambda \backslash N) \simeq\left(C_{\mathrm{Aff}(N)} \Lambda\right) / \Lambda
$$

where, $\operatorname{Aff}^{0}(\Lambda \backslash N) \subset \operatorname{Aff}(\Lambda \backslash N)$ denotes the identity component. Also, $\Lambda \cap N_{L} \subset$ $N_{L}$ is cocompact. Thus, by Malcev's theorem (3.7),

$$
\text { (4.1.3) } C_{\mathrm{Aff}(N)} \Lambda \subset C_{\mathrm{Aff}(N)} N_{L} \text {. }
$$

Moreover,

$$
N_{R}=C_{\mathrm{Aff}(N)} N_{L}=\left\{\left(t, A d_{t^{-1}}\right)\right\}
$$

is just an isomorphic copy of $N$ acting by right translations.

The identification $N_{R}=C_{\mathrm{Aff}(N)} N_{L}$ depends only on the affine structure of the affine homogeneous space, $\left(N, \nabla^{\text {can }}\right)$; compare Remark 3.1. However, an explicit isomorphism, $N_{L} \simeq N_{R}$, or equivalently the representation $N_{R}=$ $\left(t, A d_{t^{-1}}\right)$, does depend on a choice of base point (which can then be viewed as the identity element, $e \in N$ ). More generally, corresponding to each normal subgroup, $B_{L} \subset N_{L}$, there is a well-defined isomorphic subgroup, $B_{R} \subset N_{R}$. Again, a specific isomorphism, $B_{L} \simeq B_{R}$, depends on a choice of base point.

Now let $V$ be a locally defined right invariant vector field on a neighborhood $W \subset Z_{y}$. As in $\S 3$, we can find a local trivialization $\phi: U \times Z \rightarrow X$ (over a small neighborhood $U$ of $y$ ), with respect to which the affine structure on the fibres is constant. Such a trivialization is unique up to a map $U \rightarrow \operatorname{Aff}^{0}(\Lambda \backslash N)$. Since the group $\operatorname{Aff}^{0}(\Lambda \backslash N)$ is contained in $N_{R}$, it follows that this group acts trivially on local right invariant fields. Thus, $V$ has a canonical extension to $\phi(U \times W)$. In this way, we obtain a sheaf $\underline{\mathfrak{n}}$, of nilpotent Lie algebras of vector fields on $X$ and an action of the associated sheaf $\mathfrak{n}$, of simply connected nilpotent Lie groups.

The action of $G$ extends in an obvious way to an action on $\mathfrak{n}$ and the actions of $\mathfrak{n}$ and $G$ on $X$ commute in the obvious sense. In general, the action of a group on a sheaf is called locally trivial if for each open set $U$, there is a neighborhood, $W$, of the identity in $G$ such that for all $g \in W$,

$$
\rho_{g(U) \cap U, g(U)} g=\rho_{g(U) \cap U, U} \cdot
$$

Here, $\rho_{B, A}$ denotes the restriction map from $A$ to $B$.

Now the same sort of argument as was given above yields

Proposition 4.3. The action of $G$ on $\tilde{\mathfrak{n}}$ is locally trivial.

In case $G$ acts freely, it follows directly from Proposition 4.3 that there is an induced sheaf, $\tilde{\mathfrak{n}}$, on $X / G$ (see $\S 8$ for the detailed discussion).

We now discuss the quantitative behavior of the local right invariant fields constructed above. This requires a more explicit description of a local trivialization in which the affine structure on the fibres is constant.

Let $V$ be a tubular neighborhood of a fixed fibre, $Z_{y}$, such that the normal exponential map of $Z_{y}$ provides a local trivialization $\phi: U \times Z_{y} \rightarrow V$ (where $\phi\left(U \times Z_{y}\right)$ is the union of all fibres contained in $V$ ). By the proof 
of Proposition A2.2, the normal injectivity radius of $Z_{y}$ is bounded below by $c(n, A) \min (l, d(y, \partial Y))$.

Let $p_{y^{\prime}}=\phi\left(\left(y^{\prime}, z\right)\right) \in Z_{y^{\prime}}$. The universal covering space, $\left(\widehat{V}, \hat{p}_{y}\right)$, is fibred by universal covering spaces $\left(\widehat{Z}_{y^{\prime}}, \widehat{p}_{y^{\prime}}\right)$. The covering groups of all of these spaces are canonically isomorphic to $\Lambda$. Let $\operatorname{Aff}\left(\hat{Z}_{y^{\prime}}, \widehat{\nabla}\right)$ denote a group of affine automorphisms of $\widehat{Z}_{y^{\prime}}$, with respect to its canonical flat affine connection, $\hat{\nabla}$. Let $N_{L}\left(\hat{Z}_{y^{\prime}}\right) \subset \operatorname{Aff}\left(\hat{Z}_{y^{\prime}}, \widehat{\nabla}\right)$ be the corresponding canonically defined subgroup. Then (up to natural isomorphism), for all $y^{\prime}$, we can regard $\Lambda \subset \operatorname{Aff}\left(\hat{Z}_{y^{\prime}}, \widehat{\nabla}\right)$.

By Malcev's theorem (3.7), there is a unique affine equivalence $\psi_{y^{\prime}}: \hat{Z}_{y} \rightarrow$ $\widehat{Z}_{y^{\prime}}$, such that

(4.4.1) $\psi_{y^{\prime}}\left(\widehat{p}_{y}\right)=\widehat{p}_{y^{\prime}}$,

and for all $\lambda \in N_{L}\left(\hat{Z}_{y}\right)$ (or equivalently for all $\lambda \in \Lambda \cap N_{L}\left(\hat{Z}_{y^{\prime}}\right)$ ),

(4.4.2) $\psi_{y^{\prime}} \lambda=\lambda \psi_{y^{\prime}}$.

Given that such an affine equivalence exists, it is explicitly determined as follows.

By integrating the left invariant fields (i.e., the parallel fields for $\nabla^{*}$ ) we obtain the group $N_{R}\left(\widehat{Z}_{y^{\prime}}\right)$, and hence, the right invariant vector fields. By integrating these, we obtain the group $N_{L}\left(\widehat{Z}_{y^{\prime}}\right)$.

Fix $\lambda \in \Lambda \cap N_{L}\left(\widehat{Z}_{y}\right) \simeq \Lambda \cap N_{L}\left(\widehat{Z}_{y^{\prime}}\right)$. For each $y^{\prime}$, there is a unique integral curve, $c_{y^{\prime}, \lambda}$, of a certain right invariant vector field on $\hat{Z}_{y^{\prime}}$, such that $c_{y^{\prime}, \lambda}(0)=$ $\widehat{p}_{y^{\prime}}, c_{y^{\prime}, \lambda}\left(t_{\lambda}\right)=c_{y^{\prime}, \lambda}\left(t_{\lambda}\right)=\lambda\left(\widehat{p}_{y^{\prime}}\right)$. Here $t_{\lambda}$ is independent of $y^{\prime}$. Since $\psi_{y^{\prime}}$ is an affine equivalence satisfying (4.4.1), (4.4.2), we get

$$
d \psi_{y^{\prime}}\left(c_{y, \lambda}^{\prime}(0)\right)=c_{y^{\prime}, \lambda}^{\prime}(0) .
$$

Clearly, the collection of vectors, $\left\{c_{y, \lambda}^{\prime}(0)\right\}$, spans the tangent space at $p_{y}$. Thus, (4.5) determines the linear map, $d \psi_{y^{\prime}}$. Then $\psi_{y^{\prime}}$ itself is determined by the condition that it map a given right invariant field on $\widehat{Z}_{y}$ to the right invariant field on $\widehat{Z}_{y^{\prime}}$ to which it corresponds under $d \psi_{y^{\prime}}$.

Now for all $y$ we have inj $\operatorname{rad}_{\hat{p}_{y}} \geq c(n)>0$; see [BK, Proposition 4.6.3]. On the other hand, the points $\lambda(y)$ are $c(n, A) \delta$-dense in $\widehat{Z}_{y}^{m}$. Thus, we can find $\lambda_{1}, \ldots, \lambda_{m}$ such that

$$
\left|\Varangle\left(c_{y, \lambda_{i_{1}}}^{\prime}(0), c_{y, \lambda_{i_{2}}}^{\prime}(0)\right)-\frac{\pi}{2}\right| \leq c(n, A) \delta .
$$

From the preceding explicit description of $\psi_{y^{\prime}}$, together with Proposition 3.6 and standard bounds on the local trivialization, $\phi$, we readily obtain

Proposition 4.7. Let $w$ be a right invariant field on $\widehat{V} \cap B_{\hat{p}}(2 \delta)$, with $|w(\hat{p})|=1$. Then

(4.7.1) $\left|\nabla^{i} w\right| \leq c(n, A, i) l^{1-i}$. 
We now construct a canonical (and hence $G$-invariant) invariant metric for the action of $N_{L}$ on $U$, or equivalently for the action of $\mathfrak{n}$ on $X / G$ (in case $G$ acts freely). Given such a metric, it is obvious that the action of $\mathfrak{n}$ determines a nilpotent Killing structure (see Definition 1.5).

We have $\#\left(\Lambda \cap N_{L} \backslash \Lambda\right) \leq \omega_{n}$. Thus, it is clear that we can reduce to the case $\Lambda \subset N_{L}$.

Let $v$ be a tangent vector at $\hat{p} \in \widehat{V}$ and let $\langle$,$\rangle denote the pullback to$ $\widehat{V}$, of the original metric on $V$. Let $h \in N_{L}$ and let $h v$ denote the image of $v$ under the differential of $h$. Then, the function, $h \rightarrow\langle h v, h v\rangle$, is constant on the left cosets of $\Lambda$. Since the group $N_{L}$ is nilpotent, it is unimodular. Therefore, the space $\Lambda \backslash N$ inherits a canonical invariant measure $d \mu$, of total volume 1. The metric,

$$
(v, v)=\int_{N_{L} / \Lambda}\langle h v, h v\rangle d \mu,
$$

is invariant under $N_{L}$ and pushes down to the required metric on $V$.

Clearly, our construction is independent of the choice of $U$ and of the choice of base point used to define $\widehat{V}$. Thus it gives a canonical (and hence $G$ invariant) metric on $X$, which is invariant for the nilpotent Killing structure.

Proposition 4.9. The original metric, $\langle$,$\rangle , and invariant metric, ( , ), sat-$ isfy

(4.9.1) $\left|\nabla^{i}(\langle\rangle-,()),\right| \leq c(n, A, i) \delta l^{-(1+i)}$.

Proof. The estimates on left multiplication that follow immediately from (4.7.1) yield (4.9.1).

Remark 4.10. Note that the right-hand side of (4.9.1) is small provided $\delta$ is small relative to $l^{i+1}$.

Remark 4.11. One can also construct an equivariant right action on $X$; it gives rise to the nilpotent collapsing structure. The construction, which will be carried out elsewhere, does make use of the invariant metrics on fibres.

\section{The NILPOTENT KILling STRUCTURE AND INVARIANT ROUND METRIC}

\section{LOCAL FIBRATION OF THE FRAME BUNDLE}

In this section we begin the construction of the nilpotent Killing structure by constructing local fibrations of the frame bundle.

Let $M^{n}$ be a complete $A$-regular Riemannian manifold. A standard computation shows that the frame bundle, $F M^{n}$, with its natural metric, is $B$-regular (for $B=B(A)$ ).

For fixed $n$ and $A$, put

$$
\text { (5.5.1) } \mathfrak{F}=\left\{F B_{p}(2) \mid M^{n} \text { is } A \text {-regular }\right\} \text {. }
$$

Note, on the right-hand side of (5.1.1), $M^{n}$ is not fixed. (Also, we could replace 2 by any fixed $R>0$ in (5.1.1)). Let $\mathfrak{C F}$ denote the closure of $\mathfrak{F}$ with respect to the $O(n)$-Hausdorff distance, $d_{H}$. Then by [F3], $\mathfrak{C F}$ consists of $B(n, A)$ regular manifolds, $Y^{i}$. It will be convenient to assume that $A$ is normalized 
such that $A_{0}, B_{0}(n, A) \leq 1$. The induced action of $O(n)$ on $Y^{i}$ is $D(n, A)$ regular but need not be free.

Put

(5.1.2) $\mathfrak{C F}_{j}:=\left\{Y^{j} \in \mathfrak{C F} \mid \operatorname{dim} Y^{j}=j\right\}$.

Then $\mathfrak{C F}=\bigcup_{j} \mathfrak{C F}_{j}$ determines a stratification of $\mathfrak{C F}$. This fact, although we do not use it explicitly, puts our constructions in a natural context.

Clearly, $\mathfrak{C F}_{j}$ is empty for $j>n+\frac{n(n-1)}{2}$. One can also show that $\mathfrak{C F}_{j}$ is empty for $j<\frac{n(n-1)}{2}=\operatorname{dim} O(n)$; see Appendix 1. Again, we do not use this.

It follows from [GLP, §8] (together with [CGT, Theorem 4.3]) that there is a positive function, $\phi(\delta, n)$, with $\phi(\delta, n) / \delta$ increasing, such that if

(5.2.1) $Y_{1}^{j_{1}} \in \mathfrak{C} F_{j_{1}}$,

(5.2.2) inj $\operatorname{rad}_{y} \leq \min \left(\phi(\delta, n), d\left(y, \partial Y_{1}^{j_{1}}\right)\right.$, for some $y \in Y_{1}^{j_{1}}$,

then there exists $Y_{2}^{j_{2}} \in \mathfrak{C F}_{j_{2}}$ with

(5.2.3) $j_{2}<j_{1}$,

(5.2.4) $d_{H}\left(Y_{1}^{j_{1}}, Y_{2}^{j_{2}}\right)<\delta$.

From now on we suppress the dependence of $\phi$ on $n$.

Put $l_{0}=1$. Let $l_{0}>l_{1}>\cdots>l_{n+[n(n+1)] / 2}$ and $1>\delta_{0}>\delta_{1}>\cdots>$ $\delta_{n+[n(n+1)] / 2}$, be positive sequences, such that for $j \geq 1$,

(5.3.1) $\phi^{-1}\left(l_{j}\right)+\delta_{j} \leq \delta_{j-1}$.

Relation (5.3.1) can be satisfied by taking

(5.3.2) $l_{j}=\phi\left(\frac{1}{2} \delta_{j-1}\right)$,

(5.3.3) $\delta_{j} \leq \frac{1}{2} \delta_{j-1}$.

In this and subsequent sections it will be necessary to assume that $\delta_{j}$ is small enough relative to $l_{j}$ such that certain additional conditions are satisfied.

Proposition 5.4. Let $F B_{p}(1) \in \mathfrak{F}$ and let $j$ be the smallest number such that there exists $Y^{j} \in \mathfrak{C F}_{j}$ with

(5.4.1) $d_{H}\left(F B_{p}(1), Y^{j}\right) \leq \delta_{j}$.

Then for any such $Y^{j}$ and $y \in Y^{j}$

(5.4.2) inj $\operatorname{rad}_{y} \geq \min \left(l_{j}, d\left(y, \partial Y^{j}\right)\right)$.

Proof. Note that since $d_{H}\left(F B_{p}(1), F B_{p}(1)\right)=0$, the set of $Y^{j}$ satisfying (5.4.1) is nonempty. If $(5.4 .2)$ failed to hold, then by the definition of the function $\phi$, we would have

(5.5.1) $d_{H}\left(Y^{j}, Y_{1}^{j_{1}}\right) \leq \frac{1}{2} \delta_{j-1}$,

for some $Y_{1}^{j_{1}}$ with $j_{1} \leq j-1$. Then by (5.3.1) and (5.4.1), it follows that

$$
\begin{aligned}
d_{H}\left(F B_{p}(1), Y_{1}^{j_{1}}\right) & \leq d_{H}\left(F B_{p}(1), Y^{j}\right)+d_{H}\left(Y^{j}, Y_{1}^{j_{1}}\right) \\
& \leq \delta_{j}+\phi^{-1}\left(l_{j}\right) \leq \delta_{j-1} \leq \delta_{j}
\end{aligned}
$$

which is a contradiction. 
Fix $\lambda=\lambda(n)<1$ to be determined in $\S \S 6,7$.

Proposition 5.6. Let $p_{s} \in M^{n}, s=1,2$, with $j_{1} \leq j_{2}$. Let $Y_{s}^{j_{s}}$ satisfy (5.4.1) with $j_{s}$ minimal for $p_{s}$. Then there exist

(5.6.1) $f_{s}: F B_{p_{s}}(1) \rightarrow Y_{s}^{j_{s}}$,

(5.6.2) $f_{1,2}: f_{2}\left(F B_{p_{1}}(1) \cap F B_{p_{2}}(1)\right) \rightarrow Y_{1}^{j_{1}}$,

such that

(5.6.3) $f_{s}$ satisfies (2.6.1)-(2.6.6) with $\imath=l_{j_{s}}, \delta=\delta_{j_{1}}, \lambda=\lambda(n)$;

(5.6.4) $f_{1,2}$ satisfies (2.6.1)-(2.6.7) with $\lambda=l_{j_{2}}^{-1}, \delta=c(n, A) \delta_{j_{1}}^{1 / 2}$;

(5.6.5) $d\left(f_{1,2} f_{2}, f_{1}\right) \leq c(n, A) \lambda l_{j_{1}}$;

(5.6.6) $\left|\nabla\left(f_{1,2} f_{2}\right)-\nabla f_{1}\right| \leq c(n, A) \lambda$.

Proof. The existence of the fibrations, $f_{s}$, satisfying $(5.6 .1),(5.6 .3)$ follows from (5.4.1), (5.4.2).

Using (2.4.3), (2.4.4), we construct an $O(n)$-equivariant Hausdorff approximation, $h$, with domain $f_{2}\left(F B_{p_{1}}(1) \cap F B_{p_{2}}(1)\right)$ and range in $Y_{1}^{j_{1}}$. By regularizing $h$, we obtain $f_{1,2}$ satisfying (5.6.2), (5.6.4), and (5.6.5). Finally, (5.6.6) follows with the help of Proposition 2.30.

Remark 5.7. The sets $F B_{p_{1}}(1) \cap F B_{p_{2}}(1)$ are not necessarily of the form $f_{1}^{-1}\left(U_{1}\right)$ or $\left(f_{12} f_{2}\right)^{-1}\left(U_{2}\right)$ and hence, are not unions of compact fibres. To obtain actual fibrations we must restrict the domain of a map, $f_{s}$, to the set consisting of all compact fibres whose intersection with $F B_{p_{s}}\left(\frac{1}{2}\right)$ is nonempty. This is a slightly smaller set. We will deal with this (minor) point when it arises in the proof of Proposition 6.1. But in the meantime, to simplify notation, we will continue to refer to "the fibration $f_{s}$." More importantly, the maps, $f_{1}, f_{2}$ are not necessarily compatible in the sense that the fibres of $f_{2}$ need not be unions of the fibres of $f_{1}$. Equivalently, $f_{1,2} f_{2} \neq f_{1}$ in general. However, by (5.6.5) and (5.6.6), $f_{1}, f_{2}$ are almost compatible. This together with the results of Appendix 2, will be used in $\S 6$ to construct a collection of local fibrations of $F M^{n}$ that are compatible in the above sense.

Remark 5.8. The smaller the numbers, $\delta_{j}$, the more difficult the condition

$$
d_{H}\left(F B_{p}(2), Y^{j}\right) \leq \delta_{j}
$$

is to satisfy. In particular, the subsets of elements of $\mathfrak{F}$, for which there exists a nontrivial fibration also gets smaller.

Remark 5.9. If we fix $\varepsilon=1$ in Theorems 1.3 and 1.7 then we can work with a fixed sequence that is small enough for the arguments of subsequent sections to go through. But if we let $\varepsilon \rightarrow 0$, then necessarily $\delta_{j} \rightarrow 0$ as well. As a consequence, for $\varepsilon$ very small, our structure will be nontrivial only on the part of $M^{n}$ that is very collapsed. 


\section{MAKING THE LOCAL FIBRATIONS COMPATIBLE}

Let $M^{n}$ be as in $\S 5$.

The fibrations constructed in this section will be obtained by slightly modifying those constructed in $\S 5$ and restricting their domains. After this has been done, to simplify notation, we will continue to denote the modified fibrations by $f_{s}, f_{s, t}$ and their base spaces by $Y_{s}^{j_{s}}$.

Let $\lambda=\lambda(n)<1$ be a sufficiently small constant. The constraints on $\lambda(n)$ will be determined in the course of the proof of Proposition 6.1. These and the constraints entailed in the analogous constructions of $\S 7$ allow us to fix the values of $\lambda(n)$. We will assume without further mention at the end of $\S 7$ that this has been done.

Let $b:[0,1] \rightarrow[0,1]$ be an increasing function, with $b(u) \leq u$.

Proposition 6.1. Given $b$, there exists a decreasing sequence, $l_{j}=l_{j}(b, n, A)$, such that the following holds. There is a covering, $M^{n}=\bigcup_{s} B_{p_{s}}\left(\frac{1}{16}\right)$, and $O(n)$ equivariant fibrations,

(6.1.1) $f_{s}: F B_{p_{s}}\left(\frac{1}{2}\right) \rightarrow Y_{s}^{j_{s}}$,

such that for $y \in Y_{s}^{j_{s}}$,

(6.1.2) inj $\operatorname{rad}_{y} \geq \min \left(l_{j_{s}}, d\left(y, \partial Y_{s}^{j_{s}}\right)\right)$.

Moreover, if $B_{p_{s}}\left(\frac{1}{2}\right) \cap B_{p_{t}}\left(\frac{1}{2}\right) \neq \varnothing, j_{s} \leq j_{t}$, then there is an $O(n)$-equivariant fibration,

$$
f_{s, t}: f_{t}\left(F B_{p_{s}}\left(\frac{1}{2}\right) \cap F B_{p_{t}}\left(\frac{1}{2}\right)\right) \rightarrow f_{s}\left(F B_{p_{s}}\left(\frac{1}{2}\right) \cap F B_{p_{t}}\left(\frac{1}{2}\right)\right),
$$

such that

(6.1.4) $f_{s, t} f_{t}=f_{s}$.

The fibrations, $f_{s}$, satisfy:

(6.1.6) $\quad f_{s}$ is a $c(n, A) \lambda$-almost Riemannian submersion.

(6.1.7) $f_{s}$ is $\left\{C_{i}(n, A) l_{j_{s}}^{1-i}\right\}$-regular.

(6.1.8) $\quad\left|I I_{f_{s}^{-1}(y)}\right| \leq c(n, A) l_{j_{s}}^{-1}$.

(6.1.9) The maps $f_{s, t}$ satisfy (6.1.5)-(6.1.8).

(6.1.10) The (compact) fibres, $f_{s}^{-1}(y), f_{s, t}^{-1}(y)$ are diffeomorphic to nilmanifolds.

Proof. The fact that the fibres, $f_{s}^{-1}(y), f_{s, t}^{-1}(y)$ are diffeomorphic to nilmanifolds (and not just infranilmanifolds) was mentioned in the introduction and is explained further in $\S 7$ and Appendix 1.

Pick a maximal collection of points, $p_{s}$, such that for all $s, t$, (6.2.1) $d\left(p_{s}, p_{t}\right) \geq \frac{1}{64}$. 
In particular,

(6.2.2) $M^{n}=\bigcup_{s} B_{p_{s}}\left(\frac{1}{16}\right)$.

Fix a decreasing sequence,

(6.3.1) $\delta_{0}>\delta_{1}>\cdots>\delta_{n+[n(n+1)] / 2}$,

to be determined later. As in (5.3.2), define

(6.3.2) $l_{j}=\phi\left(\frac{1}{2} \delta_{j-1}\right)$.

Relative to the sequence $\left\{\delta_{j}\right\}$, choose for each $p_{s}$, a fibration,

(6.3.3) $f_{s}: F B_{p_{s}}(1) \rightarrow Y_{s}^{j_{s}}$,

satisfying (5.4.1) with $j_{s}$ minimal. Let the corresponding fibrations, $f_{s, t}$ be as in Proposition 5.6. We can assume that $\left\{\delta_{j}\right\}$ is such that (6.1.9) holds.

In order to make it clear that when we repeatedly modify our fibrations, approximately compatible fibrations do not eventually become too far apart, we use a technical device.

As in Lemma 2.2 of [CG1], we partition the set $\left\{p_{s}\right\}$ into disjoint subsets $S_{1}, \ldots, S_{N(n)}$, such that if $p_{s}, p_{u} \in S_{k}$, then

(6.4.1) $d\left(p_{s}, p_{u}\right)>4$.

In particular, those balls, $B_{p_{t}}(1)$, whose intersection with a fixed ball, $B_{p_{s}}(1)$, is nonempty, all belong to different subsets, $S_{k}$. Thus, there are at most $N(n)$ such balls.

Put

(6.4.2) $S_{k, j}=\left\{p_{s} \in S_{k} \mid j_{s}=j\right\}$.

There are $T(n)=N(n)\left(n+1+\frac{n(n+1)}{2}\right)$ of the $S_{k, j}$, some of which might be empty. Put

(6.4.3) $S_{k, j}=S^{k+N(n) j}$.

Note that if $S^{\alpha}=S_{k(\alpha), j(\alpha)}$, then

(6.4.4) $\alpha<\alpha^{\prime}$ implies $j(\alpha) \leq j\left(\alpha^{\prime}\right)$.

Also, $p_{s} \in S^{\alpha}, f_{s}: B_{p_{s}}(1) \rightarrow Y_{s}^{j_{s}}$, implies

(6.4.5) $j_{s}=j(\alpha)$.

In order to make our fibrations compatible, we now modify them in a total of $T(n)$-stages, one for each $S^{\alpha}$. Each stage, say $\alpha_{0}$, is divided into $\left(2^{T(n)-\alpha_{0}-1}\right)$ steps, one for each nonempty subset, $\left(\alpha_{1}, \ldots, \alpha_{m}\right)$, with $\alpha_{0}<\alpha_{1}<\cdots<$ $\alpha_{m} \leq T(n)$. Thus, there are $N^{\prime}(n)=2^{T(n)-1}-(T(n)-1)$ steps in all. (The order in which the steps are performed is specified below.)

At a given step we must also decrease the radii of the balls involved by a definite amount. The notation is simplest if at the end of each step $\alpha_{0}$, we actually decrease the radii of all balls (i.e., with centers $p_{1}, p_{2}, p_{3}, \ldots$ ) by an amount $\frac{1}{2 N^{\prime}(n)}$. Thus, at the beginning of a given step, every ball has radius, $r=1-\frac{1}{2 N^{\prime}(n)} \times$ the number of steps already peformed. 
Note that since at each stage we decrease the radii of our balls by exactly $\frac{1}{2 N^{\prime}(n)}$ we certainly want $\lambda=\lambda(n)<\frac{1}{2 N^{\prime}(n)}$.

At step $\left(\alpha_{1}, \ldots, \alpha_{m}\right)$ of stage $\alpha_{0}$, we modify only the fibrations $f_{i_{l}}, 1 \leq l \leq$ $m$, and $f_{i_{l_{1}}, i_{l_{2}}}, 1 \leq l_{1}<l_{2} \leq m$, over sets of the form $F B_{p_{i_{0}}}(r) \cap \cdots \cap F B_{p_{i_{m}}}(r)$ (respectively $\left.f_{i_{l_{2}}}\left(F B_{p_{i_{0}}}(r) \cap \cdots \cap F B_{p_{i_{m}}}(r)\right)\right)$ where $p_{i_{l}} \in S^{\alpha_{l}}$. Note that for certain $\left(\alpha_{1}, \ldots, \alpha_{m}\right)$ (e.g., unless $S^{\alpha_{0}}, \ldots, S^{\alpha_{m}}$ all belong to distinct $S_{u}$ ) there will be no such nonempty intersections. However, if at any step there are no nonempty intersections, we simply decrease the radii of all balls by $\frac{1}{2 N^{\prime}(n)}$ and proceed to the next step.

Now we can explain the reason for introducing the sets $S_{u}$. If $\hat{p}_{i_{l}} \in S^{\alpha_{l}}$ and $\left(\hat{p}_{i_{0}}, \ldots, \hat{p}_{i_{m}}\right)$ is distinct from $\left(p_{i_{0}}, \ldots, p_{i_{m}}\right)$ then (by construction)

$$
\left(F B_{p_{i_{0}}}(r) \cap \cdots \cap F B_{p_{i_{m}}}(r)\right) \cap\left(F B_{\hat{p}_{i_{0}}}(r) \cap \cdots \cap F B_{\hat{p}_{i_{m}}}(r)\right)=\varnothing .
$$

This guarantees that the various modifications performed at step $\left(\alpha_{1}, \ldots, \alpha_{m}\right)$ of stage $\alpha_{0}$ do not interact with one another (and that a given fibration is modified at most $N^{\prime}(n)$ times). As a consequence, fibrations that are initially almost compatible do not grow uncontrollably further apart as the construction progresses.

It is important that in carrying out the modifications, the stages are arranged in descending order; i.e., we start with stage $T(n)$, then pass to stage $T(n)-1$, etc. It is also important that the steps of stages $\alpha_{0}$ are arranged as follows. First we do step $\left(\alpha_{0}+1, \alpha_{0}+2, \ldots, T(n)\right)$. Then, in some (arbitrary) order, we do the steps corresponding to subsets of $\left(\alpha_{1}, \ldots, \alpha_{m}\right)$ of cardinality, $T(n)-$ $\alpha_{0}-1$; then, in some (arbitrary) order, the steps corresponding to subsets of cardinality $T(n)-\alpha_{0}-2$, etc.

Let $r$ be the common radius of all balls at the beginning of step $\left(\alpha_{1}, \ldots, \alpha_{m}\right)$ of stage $\alpha_{0}$. At the beginning of this step, we can assume by induction that the following holds.

Let $\alpha_{0}^{\prime} \geq \alpha_{0}$ and let $\left(\alpha_{1}^{\prime}, \ldots, \alpha_{m^{\prime}}^{\prime}\right)$ be a step of stage $\alpha_{0}^{\prime}$ that has already been completed (automatic unless $\alpha_{0}^{\prime}=\alpha_{0}, m^{\prime} \leq m$ ). Let $\underline{p}_{i_{l^{\prime}}} \in S^{\alpha_{l^{\prime}}^{\prime}}, 0 \leq l^{\prime} \leq$ $m^{\prime}$. Then for $0 \leq l_{1}^{\prime}<l_{2}^{\prime} \leq m^{\prime}$, our (previously redefined) fibrations satisfy (6.6.1) $f_{i_{l_{1}^{\prime}} i_{l_{2}^{\prime}}} f_{i_{l_{2}^{\prime}}}=f_{i_{l_{1}^{\prime}}}$ on $F B_{\underline{p}_{i_{0}}}(r) \cap \cdots \cap F B_{\underline{p}_{i_{m}}}(r)$. $j_{u}$,

In addition, we can assume by induction that for all $s, t, u$ with $j_{s} \leq j_{t} \leq$

(6.6.2) $\operatorname{dia}\left(f_{s}^{-1}(y)\right), \operatorname{dia}\left(f_{s, t}^{-1}(y)\right) \leq c(n, A) \delta_{j_{s}}$.

(6.6.3) $f_{s}, f_{s, t}$ are $c(n, A) \lambda$-almost Riemannian submersions.

(6.6.4) $f_{s}, f_{s, t}$ are $\left\{C_{i}(n, A) l_{j_{s}}^{-1}\right\}$-regular.

(6.6.5) $d\left(f_{s, t} f_{t}, f_{s}\right) \leq c(n, A) \lambda l_{j_{s}}$.

(6.6.6) $\left|\nabla\left(f_{s, t} f_{t}\right)-\nabla f_{s}\right| \leq c(n, A) \lambda$.

(6.6.7) $d\left(f_{s, t} f_{t, u}, f_{s, u}\right) \leq c(n, A) \lambda l_{j_{s}}$.

(6.6.8) $\left|\nabla\left(f_{s, t} f_{t, u}\right)-\nabla f_{s, u}\right| \leq c(n, A) \lambda$. 
We now define certain $O(n)$-equivariant self-diffeomorphisms

(6.7.1) $\psi_{i_{l}}: F B_{p_{i_{l}}}(r) \rightarrow F B_{p_{i_{l}}}(r), \quad 1 \leq l \leq m$

and

$$
\xi_{i_{l}}: f_{i_{l}}\left(F B_{p_{i_{l}}}(r)\right) \rightarrow f_{i_{l}}\left(F B_{p_{i_{l}}}(r)\right), \quad 1 \leq l \leq m
$$

(We suppress the dependence of these maps on $\left(\alpha_{0}, \ldots, \alpha_{m}\right)$.) Eventually, we will redefine $f_{i}$ to be

(6.7.3) $\xi_{i_{l}}^{-1} f_{i_{l}} \psi_{i_{l}}, \quad 1 \leq l \leq m$,

and redefine $f_{i_{l_{1}}, i_{l_{2}}}$ to be

$$
\xi_{i_{l_{1}}}^{-1} f_{i_{l_{1}}, i_{l_{2}}} \xi_{i_{l_{2}}}, \quad 1 \leq l_{1}<l_{2} \leq m \text {. }
$$

Until this is done explicitly, $f_{i_{l}}, f_{i_{l_{l}}, i_{l_{2}}}$ retain their previous meanings.

We now use Proposition A2.2 to construct the diffeomorphism $\psi_{i_{l}}$. (Remarks similar to those that follow also apply to the construction of the diffeomorphism $\xi_{i_{l}}$ below). Since Proposition A2.2 holds for fibrations with compact fibres, we first restrict the map $f_{i_{0}}$ (which is used in defining the various $\psi_{i_{l}}$ ) to the subset of $F B_{p_{i_{0}}}(r) \cap \cdots \cap F B_{p_{i_{m}}}(r)$ consisting of the union of all compact fibres of $f_{i_{0}}$. The set contains $\left(F B_{p_{i_{0}}}(r) \cap \cdots \cap F B_{p_{i_{m}}}(r)\right)_{c(n, A) \delta_{j\left(\alpha_{0}\right)}}$ (the notation is as in (2.3)).

In view of (6.6.3)-(6.6.6), by Proposition A.2.2 we can find an $O(n)$-equivariant map, $\psi\left(=\psi_{i_{l_{1}}, \ldots, i_{l_{m}}}\right)$ such that:

(6.8.1) $\psi$ is a self-diffeomorphism of $F B_{p_{i_{0}}}(r) \cap \cdots \cap F B_{p_{i_{m}}}(r)$.

(6.8.2) $\psi$ is the identity near the boundary.

(6.8.3) $f_{i_{0}, i_{1}}\left(f_{i_{1}} \psi\right)=f_{i_{0}}$ on $F B_{p_{i_{0}}}\left(r-\frac{1}{2 N^{\prime}(n)}\right) \cap \cdots \cap F B_{p_{i_{m}}}\left(r-\frac{1}{2 N^{\prime}(n)}\right)$.

(6.8.4) $\psi$ is the identity on the subset of

$$
F B_{p_{i_{0}}}\left(r-\frac{1}{2 N^{\prime}(n)}\right) \cap \cdots \cap F B_{p_{i_{m}}}\left(r-\frac{1}{2 N^{\prime}(n)}\right)
$$

on which $f_{i_{0}, i_{1}} f_{i_{1}}=f_{i_{0}}$.

(6.8.5) $d(\psi$, Ident $) \leq c(n, A) \lambda l_{j\left(\alpha_{0}\right)}$,

(6.8.6) $\mid \nabla \psi-$ Ident $\mid \leq c(n, A) \lambda$.

(6.8.7) $\psi$ is $\left\{C_{i}(n, A) l_{j\left(\alpha_{1}\right)}^{1-i}\right\}$-regular.

Define $\psi_{i}$ as in (6.7.1) by

$$
\psi_{i_{l}}(x)= \begin{cases}\psi(x) & x \in F B_{p_{i_{0}}}(r) \cap \cdots \cap F B_{p_{i_{m}}}(r), \\ x & x \notin F B_{p_{i_{0}}}(r) \cap \cdots \cap F B_{p_{i_{m}}}(r) .\end{cases}
$$

We now define the diffeomorphisms, $\xi_{i_{l}}$.

In view of (6.6.3), (6.6.4), (6.6.7), (6.6.8), by Proposition A2.2, we can find an $O(n)$-equivariant map, $\xi_{i_{l}}(l \geq 2)$ such that:

(6.9.1) $\xi_{i_{l}}$ is a self-diffeomorphism of $f_{i_{l}}\left(F B_{p_{i_{0}}}(r) \cap \cdots \cap F B_{p_{i_{m}}}(r)\right)$. 
(6.9.2) $\xi_{i_{1}}$ is the identity near the boundary.

(6.9.3) $f_{i_{0}, i_{1}} f_{i_{1}, i_{l}} \xi_{i_{l}}=f_{i_{0}, i_{l}}$ on $f_{i_{l}}\left(F B_{p_{i_{0}}}\left(r-\frac{1}{2 N^{\prime}(n)}\right) \cap \cdots \cap F B_{p_{i_{m}}}\left(r-\frac{1}{2 N^{\prime}(n)}\right)\right)$.

(6.9.4) $\xi_{i_{l}}$ is the identity on the subset of

$$
f_{i_{l}}\left(F B_{p_{i_{0}}}\left(r-\frac{1}{2 N^{\prime}(n)}\right) \cap \cdots \cap F B_{p_{i_{m}}}\left(r-\frac{1}{2 N^{\prime}(n)}\right)\right)
$$

on which $f_{i_{0}, i_{1}} f_{i_{1}, i_{l}}=f_{i_{0}, i_{l}}$.

(6.9.5) $d\left(\xi_{i_{l}}\right.$, Ident $) \leq c(n, A) \lambda l_{j\left(\alpha_{0}\right)}$.

(6.9.6) $\mid \nabla \xi_{i_{l}}$ - Ident $\mid \leq c(n, A) \lambda$.

(6.9.7) $\xi_{i_{l}}$ is $\left\{C_{i}(n, A) l_{j\left(\alpha_{1}\right)}^{1-i}\right\}$-regular.

Extend $\xi_{i_{l}}$ to all of $f_{i_{l}}\left(F B_{p_{i_{l}}}(r)\right)$ by defining it to be the identity map off $f_{i_{l}}\left(F B_{p_{i_{0}}}(r) \cap \cdots \cap F B_{p_{i_{m}}}(r)\right)$. Also define $\xi_{i_{1}}$ to be the identity map.

We now examine the effect of modifying $f_{i_{l}}, f_{i_{l_{1}}, i_{l_{2}}}$ as in (6.7.3), (6.7.4) on our induction hypotheses.

First of all, it follows from (6.6.1), (6.8.4), (6.9.4) that $\psi_{i_{l}}, \xi_{i_{l}}$ is equal to the identity over the subset of $F B_{p_{i_{0}}}(r) \cap \cdots \cap F B_{p_{i_{l}}}(r)$ that intersects any $F B_{p_{i}}(r)$, where $p_{i} \in S^{\beta}, \beta>\alpha_{0}, \beta \neq \alpha_{1}, \ldots, \alpha_{m}$. Moreover, the corresponding statement holds for $\xi_{i_{1}}$. As a consequence, in examining the effect of the proposed modifications on (6.6.1), we can assume that $\alpha_{l_{u}^{\prime}}=\alpha_{l_{u}}$, for some $\alpha_{l_{u}} \in S^{\alpha_{l_{u}}}$, $u=1,2$ (since otherwise, nothing changes).

Next observe that for $1 \leq l_{1}<l_{2} \leq m$, on $F B_{p_{i_{0}}}(r) \cap \cdots \cap F B_{p_{i_{m}}}(r)$, by (6.6.1) we have

$$
\begin{aligned}
\left(\xi_{i_{l_{1}}}^{-1} f_{i_{l_{1}}, i_{l_{2}}} \xi_{i_{l_{2}}}\right)\left(\xi_{i_{l_{2}}}^{-1} f_{i_{l_{2}}} \psi_{i_{l_{2}}}\right) & =\xi_{i_{l_{1}}}^{-1} f_{i_{l_{1}}, i_{l_{2}}} f_{i_{l_{2}}} \psi \\
& =\xi_{i_{l_{1}}}^{-1} f_{i_{l_{1}}} \psi \\
& =\left(\xi_{i_{l_{1}}}^{-1} f_{i_{l_{1}}} \psi_{i_{l_{1}}}\right)
\end{aligned}
$$

(while outside $F B_{p_{i_{0}}}(r) \cap \cdots \cap F B_{p_{i_{m}}}(r)$, the maps $\psi_{l_{u}}, \xi_{l_{u}}$ are the identity).

Now by construction,

(6.10.2) $f_{i_{0}, i_{1}}\left(\xi_{i_{1}}^{-1} f_{i_{1}} \psi_{i_{1}}\right)=f_{i_{0}} \quad$ on $F B_{p_{i_{0}}}\left(r-\frac{1}{2 N^{\prime}(n)}\right) \cap \cdots \cap F B_{p_{i_{m}}}\left(r-\frac{1}{2 N^{\prime}(n)}\right)$

(recall $\xi_{i_{1}}$ is the identity map).

Finally, for $l \geq 2$, by (6.6.1), on $F B_{p_{i_{0}}}\left(r-\frac{1}{2 N^{\prime}(n)}\right) \cap \cdots \cap F B_{p_{i_{m}}}\left(r-\frac{1}{2 N^{\prime}(n)}\right)$, we have

$$
\begin{aligned}
f_{i_{0}, i_{l}}\left(\xi_{i_{l}}^{-1} f_{i_{l}} \psi_{i_{l}}\right) & =f_{i_{0}, i_{1}} f_{i_{1}, i_{l}} \xi_{i_{l}} \xi_{i_{l}}^{-1} f_{i_{l}} \psi_{i_{l}} \\
& =f_{i_{0}, i_{1}} f_{i_{1}, i_{l}} f_{i_{l}} \psi_{i_{l}} \\
& =f_{i_{0}, i_{1}} f_{i_{1}} \psi_{i_{1}} \\
& =f_{i_{0}} .
\end{aligned}
$$


Thus, if we redefine the maps $f_{i_{l}}, 1 \leq l \leq m$ and $f_{i_{l_{1}}}, f_{i_{l_{2}}}, 1 \leq l_{1}<l_{2} \leq m$ as in (6.7.3), (6.7.4), then the part of the induction hypothesis corresponding to (6.6.1) holds (here, of course, $r$ is replaced by $r-\frac{1}{2 N^{\prime}(m)}$ ). Moreover, it is straightforward to verify that the redefined maps satisfy the induction hypotheses corresponding to (6.6.2)-(6.6.7). This completes the step $\left(\alpha_{1}, \ldots, \alpha_{m}\right)$.

By taking $\lambda=\lambda(n)$ sufficiently small and each $\delta_{j}$ of our sequence sufficiently small relative to $l_{j}$, relations $(6.6)$ guarantee that when the whole modification process has been completed, the resulting maps will satisfy the conditions of Proposition 6.1. This completes the proof.

Remark 6.11. Examination of the proof of Proposition 6.1 shows why we stated Proposition A2.2 in such a way that a bound on the Hessian of only one of the maps in the proposition is required. For example, the Hessian of the map, $f_{i_{1}}$, and hence of the map, $f_{i_{0}, i_{1}} f_{i_{1}}$, used in defining $\psi$, is bounded by a constant times $l_{j\left(\alpha_{1}\right)}^{-1}$, rather than by $l_{j\left(\alpha_{0}\right)}^{-1}$, as is the case for the map $f_{i_{0}}$. Note that $l_{j\left(\alpha_{1}\right)}^{-1}>>l_{j\left(\alpha_{0}\right)}^{-1}$, if $j\left(\alpha_{0}\right)<j\left(\alpha_{1}\right)$. Also, care had to be taken in choosing the method of redefining the maps $f_{i_{1}}, f_{i_{l_{1}}, i_{l_{2}}}$, in order to ensure that control over Hessians of relevant maps was not lost in the induction process.

Remark 6.12. By $\S \S 3,4$, the fibres of our maps, $f_{s}$, carry canonical affine structures. However, the inclusions of fibres implied by (6.1.4) (namely, $f_{t}^{-1}\left(y_{t}\right) \subset$ $f_{s}^{-1}\left(y_{s}\right)$, where $\left.y_{t} \in f_{s, t}^{-1}\left(y_{s}\right)\right)$ need not be compatible with these affine structures. Arranging this is the subject of $\S 7$. However, if we pretend that it is already the case, then Proposition 6.1 summarizes much of what we aim to accomplish in this paper.

\section{MAKING THE LOCAL GROUP ACTIONS COMPATIBLE}

In this section we complete the construction of the nilpotent Killing structure on the frame bundle.

By $\S 6$ we have a mutually compatible system of maps,

$$
Z_{s} \rightarrow F B_{p_{s}}\left(\frac{1}{2}\right) \stackrel{f_{s}}{\rightarrow} Y_{s}^{j_{s}}
$$

such that $M^{n}=\bigcup_{s} B_{p_{s}}\left(\frac{1}{16}\right)$. As pointed out in the proof of Proposition 6.1, to obtain actual fibrations with compact fibres, we must replace the sets $F B_{p_{s}}\left(\frac{1}{2}\right)$ in (7.1) by slightly smaller sets, i.e., the union of all compact fibres intersecting $F B_{p_{s}}\left(\frac{1}{2}\right)$. This is to be understood (sometimes without explicit mention) in what follows. We denote by $\mathscr{F}_{s}$ the fibration corresponding to (7.1).

By $\S 3$, each fibre, $Z_{s}$, carries a canonical flat affine structure, affine isomorphic to some $\left(\Lambda_{s} \backslash N_{s}, \nabla^{\text {can }}\right)$, and a canonical metric, whose image under such an isomorphism lifts to a left invariant metric on $N_{s}$.

In our case, we actually have

$$
\Lambda_{s} \subset\left(N_{s}\right)_{L} \subset \operatorname{Aff}\left(N_{s}, \nabla^{\text {can }}\right)
$$


(and not just \#( $\left.\left.\Lambda_{s} \cap\left(N_{s}\right)_{L} \backslash \Lambda_{s}\right) \leq \omega_{n}\right)$. This follows from the fact that short closed loops on the frame bundle of an $A$-regular Riemannian manifold (in this case $Z_{s}$ ) automatically have small holonomy (compare [G, R] and Appendix 1).

If $B_{p_{s}}\left(\frac{1}{2}\right) \cap B_{p_{t}}\left(\frac{1}{2}\right)$ is nonempty, then (say) each fibre $Z_{t}$ of $\mathscr{F}_{t}$ is contained in some fibre, $Z_{s}$ of $\mathscr{F}_{s}$. However, this inclusion need not be compatible with the affine structures. We now show that $\mathscr{F}_{t}$ lies close to a unique $O(n)$-equivariant subfibration, $\mathscr{F}_{t}^{\prime}$ of $\mathscr{F}_{s}$, such that the tangent bundle to the fibres, $T Z_{t}^{\prime}$, is a totally geodesic sub-bundle of $\left(T Z_{s}, \nabla^{\text {can }}\right)$. Given this and an argument that replaces Proposition A2.2, the construction of the nilpotent Killing structure can be completed by arguments like those in $\S 6$. Specifically, we will obtain modified fibrations such that on nonempty intersections of their domains, the inclusions of fibres are compatible with affine structures. Then we construct nilpotent Killing structures and invartiant metrics as in $\S 4$.

For each fibre, $Z_{s}$ of $\mathscr{F}_{s}$, there is a fibration

$$
Z_{t} \rightarrow Z_{s} \longrightarrow W
$$

Using the fibration in (7.3) and the affine structure on $Z_{s}$, we will construct for each $Z_{s}$, a fibration,

$$
Z_{t}^{\prime} \rightarrow Z_{s} \rightarrow W^{\prime}
$$

which has totally geodesic fibres and that lies close to the one in (7.3). Then we define the fibration $\mathscr{F}_{t}^{\prime}$ to be the one whose fibres are all $Z_{t}^{\prime}$ in (7.4) (as $Z_{s}$ in (7.4) varies).

Let $\hat{Z}_{s}$ be the universal covering space of $Z_{s}$. Although a specific choice of $\hat{Z}_{s}$ is gotten by choosing a base point, the construction of $\mathscr{F}_{t}^{\prime}$ that follows will not depend on the particular choice of base point. Thus, our construction will automatically be $O(n)$-equivariant.

Write $Z_{s}=\Lambda_{s} \backslash \hat{Z}_{s}$ and let $\pi: \widehat{Z}_{s} \rightarrow Z_{s}$. The group $\pi_{1}\left(Z_{t}\right)$ does depend on a choice of base point. However, the existence of the fibration in (7.3) implies that

$$
i\left(\pi_{1}\left(Z_{t}\right)\right):=\Lambda_{t} \subset \Lambda_{s},
$$

the image of $\pi_{1}\left(Z_{t}\right)$ under the map induced by the inclusion, $Z_{t} \rightarrow Z_{s}$, is a well-defined normal subgroup.

Lemma 7.6. The map $\pi_{1}\left(Z_{t}\right) \stackrel{i}{\rightarrow} \pi_{1}\left(Z_{s}\right)$ is an injection.

Proof. Let $Z_{s}, Z_{t}$ be the fibres of $f_{s}, f_{t}$, respectively. Then $W$ is a fibre of $f_{s, t}$. Hence, by (6.1.10), $W$ is almost flat, and, in particular, aspherical. Then, by applying the homotopy sequence for fibrations to the fibration in (7.3), our claim follows.

Let $\hat{\nabla}$ denote the pullback to $\hat{Z}_{s}$, of the flat affine connection on $Z_{s}$. Then

$$
\left(\hat{Z}_{s}, \hat{\nabla}\right) \simeq\left(N_{s}, \nabla^{\text {can }}\right),
$$

where we view $N_{s}$ as an affine homogeneous space; i.e., we do not distinguish a base point. We regard the invariantly defined subgroup $\left(N_{s}\right)_{L}$ as contained 

in $\operatorname{Aff}\left(\hat{Z}_{s}, \hat{\nabla}\right)$; compare Remark 4.2. Then

$$
\Lambda_{s} \subset\left(N_{s}\right)_{L}
$$

By Malcev's theorem (3.7), there is a unique simply connected subgroup,

$$
\left(N_{t}^{\prime}\right)_{L} \subset\left(N_{s}\right)_{L}
$$

which contains $\Lambda_{t}$ as a cocompact subgroup. Since $\Lambda_{t} \subset \Lambda_{s}$ is normal, $\left(N_{t}^{\prime}\right)_{L} \subset$ $\left(N_{s}\right)_{L}$ is normal as well. Define $\Lambda_{t}^{\prime} \supseteq \Lambda_{t}$ by

(7.9.2) $\quad \Lambda_{t}^{\prime}=\Lambda_{s} \cap\left(N_{t}^{\prime}\right)_{L}$.

We will show in Lemma 7.13 that, in fact, $\Lambda_{t}^{\prime}=\Lambda_{t}$.

Let the fibration, $Z_{t}^{\prime} \rightarrow Z_{s} \rightarrow W^{\prime}$, in (7.4) be the one whose fibres are the orbits,

(7.9.3) $\quad Z_{t}^{\prime}=\Lambda_{t}^{\prime} \backslash\left(N_{t}^{\prime}\right)_{L}(z)$,

where $z \in \widehat{Z}_{s}$ (compare Remark 3.9). Then the fibration, $\mathscr{F}_{t}^{\prime}$, is as specified after (7.4). We now show that this $\mathscr{F}_{t}^{\prime}$ is close to $\mathscr{F}_{t}$ over $B_{p_{s}}\left(\frac{1}{2}\right) \cap B_{p_{t}}\left(\frac{1}{2}\right)$.

Note that Lemma 7.6 already implies

$$
\operatorname{dim} Z_{t}=\operatorname{dim} Z_{t}^{\prime}
$$

By (6.1.8),

(7.11.1) $\left|I I_{Z_{t}}\right| \leq c(n, A) l_{j_{t}}^{-1}$,

while from (3.6.1), (6.1.5), and (6.1.8) we get

(7.12.2) $\left|I I_{Z_{t}^{\prime}}\right| \leq c(n, A)\left(b\left(l_{j_{s}}\right) l_{j_{s}}^{-2}+l_{j_{s}}^{-1}\right) \leq 2 c(n, A) l_{j_{s}}^{-1}$,

(where $l_{j_{s}}^{-1}<l_{j_{t}}^{-1}$ and $j_{s}, j_{t}$ are as in $\S 6$ ).

Now suppose

(7.12.1) $b(u) \leq \theta u$,

so that

(7.12.2) $b\left(l_{j_{t}}\right) \leq \theta l_{j_{t}}$,

where $\theta=\theta(n, A)$ is a small constant. Then we obtain

Lemma 7.13. (1) There exists $c^{*}(n, A)>0$, such that the normal injectivity radius of a fibre $Z_{t}$ is bounded below by $\min \left(c^{*}(n, A) l_{j_{t}}, d\left(Z_{t}, \partial\left(\operatorname{dom} \mathscr{F}_{t}\right)\right)\right.$.

(2) If $Z_{t}, Z_{t}^{\prime}$ are fibres of $\mathscr{F}_{t}, \mathscr{F}_{t}^{\prime}$ passing through $z$, then

(7.13.1) $d\left(Z_{t}, Z_{t}^{\prime}\right) \leq \frac{1}{2} c(n, A) \theta^{2}(n, A) l_{j_{t}}$.

(3) For $\theta(n, A)$ sufficiently small, if $d(z, \partial(\operatorname{dom}(\mathscr{F})))>c^{*}(n, A) l_{j_{t}}$, then normal projection onto $Z_{t}$ defines a diffeomorphism from $Z_{t}^{\prime}$ to $Z_{t}$. In particular, $\Lambda_{t}^{\prime}=\Lambda_{t}$.

Proof. (1) The estimate on the normal injectivity radius is contained in the proof of Proposition A2.2.

(2) By Proposition 4.6.3 of [BK] there exists $c^{*}(n, A)>0$, such that

(7.14.1) inj $\operatorname{rad} Z_{t} \geq c^{*}(n, A) l_{j_{t}}$ 
induced by the invariant metric constructed in Proposition 4.9. By (4.9.1) the same holds for the metric induced by the given one.

Let $\hat{z} \in \pi^{-1}(z) \subset \widehat{Z}_{t}$ and let $\widehat{Z}_{t}, \widehat{Z}_{t}^{\prime}$ be the components of $\pi^{-1}\left(Z_{t}\right)$, $\pi^{-1}\left(Z_{t}^{\prime}\right)$ through $\hat{z}_{t}$. These have in common the $b\left(i_{j}\right)$-dense set, $\Lambda_{t}(z)$ with $b(u)$ as in (7.12). Additionally, they satisfy the bounds of (7.10), (7.11). It follows easily that the tangent spaces, $\left(\widehat{Z}_{t}\right)_{\hat{z}},\left(\widehat{Z}_{t}^{\prime}\right)_{\hat{z}}$ satisfy

(7.14.2) $\Varangle\left(\left(\widehat{Z}_{t}\right)_{\hat{z}},\left(\hat{Z}_{t}^{\prime}\right)_{\hat{z}}\right) \leq c(n, A) \theta$.

Similarly, for each $\hat{q}^{\prime} \in \widehat{Z}_{t}^{\prime}$ there is a unique closest $\hat{q} \in \widehat{Z}_{t}$, such that

(7.14.3) $d\left(\hat{q}^{\prime}, \hat{q}\right) \leq c(n, A) \theta^{2} l_{j_{t}}$.

For $\theta(n, A)$ sufficiently small, this yields (2).

(3) Finally, the angle between $\left(\widehat{Z}_{t}\right)_{\hat{q}}$ and the parallel translate of $\left(\widehat{Z}_{t}\right)_{\hat{q}^{\prime}}$ (along, unique minimal geodesic from $\hat{q}^{\prime}$ to $\hat{q}$ ) is at most $c(n, A) \theta$. It is now clear that a normal projection to $Z_{t}$ defines a covering map from $Z_{t}^{\prime}$ to $Z_{t}$. Since $\Lambda_{t} \subset \Lambda_{t}^{\prime}$, this must be a diffeomorphism.

Remark 7.15. The principle behind (2) above is the following. If two functions agree on an $\varepsilon$-dense set and have derivatives up to order $N$ bounded, then they are close to order $\varepsilon^{N-1}$, their derivatives are close to order $\varepsilon^{N-2}$, etc.

Now suppose that for $\theta=\theta\left(n, A, i_{0}\right), i_{0} \geq 1$, in fact, (7.16.1) $b(u) \leq \theta u^{1+i_{0}}$.

Then by Proposition 4.9, we obtain an $(O(n)$-invariant) invariant metric, $(, \quad)$ on dom $\mathscr{F}_{s}$ with the following property. If $\langle$,$\rangle denotes the restriction to$ dom $\mathscr{F}_{s}$ of our original metric, $g$, and $\theta\left(n, A, i_{0}\right)$ is sufficiently small, then for $i \leq i_{0}$,

(7.16.2) $\left|\nabla^{i}(\langle\rangle-,()),\right| \leq l_{j_{s}}^{i_{0}-i}$.

At this point, we can match the affine structures on $\mathscr{F}_{t}, \mathscr{F}_{t}^{\prime}$, by adapting to our situation, the center of mass argument of [GrK], used there to prove the stability of compact group actions.

Let $y \in Y^{j_{t}}$. Put $V=f_{t}^{-1}\left(B_{y}\left(\frac{1}{2} l_{j_{t}}\right)\right)$. By shrinking $V$ slightly, we obtain a subdomain $\underline{V} \subset V$ such that if $q \in \underline{V}$, then the fibres of $\mathscr{F}_{t}$ and $\mathscr{F}_{t}^{\prime}$ through $q$ are both contained in $V$. Let $(\widehat{V}, \hat{q})$ denote the universal covering space of $V$. Then we have the following preliminary result.

Lemma 7.17. There exists $c^{*}(n, A)>0$ such that for $\hat{q}_{1} \in \widehat{V}$,

(7.17.1) inj $\operatorname{rad}_{\hat{q}_{1}} \geq \min \left(c^{*}(n, A) l_{j_{s}}, d\left(\hat{q}_{1}, \partial \widehat{V}\right)\right)$.

Proof. This follows easily from (7.11.1), Lemma 7.13 (1) and (7.14).

The isomorphism, $\Lambda_{t} \simeq \Lambda_{t}^{\prime}$, extends to a canonical isomorphism $\left(N_{t}\right)_{L} \simeq$ $\left(N_{t}^{\prime}\right)_{L}$. Let $\mu_{t}, \mu_{t}^{\prime}$ denote the actions of $\left(N_{t}\right)_{L},\left(N_{t}^{\prime}\right)_{L}$. Then for all $\lambda \in \Lambda_{t} \simeq$ $\Lambda_{t}^{\prime}$, we have

(7.18.1) $\mu_{t}(\lambda)=\mu_{t}^{\prime}(\lambda)$. 
Thus, the map $\mu_{t}(h)\left(\mu_{t}^{\prime}\right)^{-1}(h)$ is well defined on $\left(N_{t}\right)_{L} / \Lambda_{t} \simeq\left(N_{t}^{\prime}\right)_{L} / \Lambda_{t}^{\prime}$. A slight variant of the argument leading to Proposition 4.7 shows that for all $h$,

$$
\left|\nabla^{i}\left(\mu_{t}(h)\left(\mu_{t}^{\prime}\right)^{-1}(h)\right)-\operatorname{Ident}\right| \leq c\left(n, A, i_{0}\right) l_{j_{s}}^{i_{0}-i}
$$

Similarly, by integrating over $\left(N_{t}\right)_{L} / \Lambda_{t} \simeq\left(N_{t}^{\prime}\right)_{L} / \Lambda_{2}^{\prime}$ (rather than all of $\left.N_{t}\right)$ we can define the center of mass of the map $\mu_{2}\left(\mu_{2}^{\prime}\right)^{-1}$; compare [GrK] and (4.8). As in [GrK], this yields an $O(n)$-equivariant diffeomorphism, $\hat{\psi}$, such that

(7.19.1) $\hat{\psi} \mu_{t}=\mu_{t}^{\prime} \hat{\psi}$,

(7.19.2) $\left|\nabla^{i} \hat{\psi}-\operatorname{Ident}\right| \leq c\left(n, A, i_{0}\right) l_{j_{s}}^{i_{0}-i}$.

It follows from (7.17) and (7.19.1) that $\hat{\psi}$ commutes with the action of $\Lambda_{t}$. Thus, the collection of maps, $\hat{\psi}$, obtained by varying $y \in Y^{j_{t}}$, induces a well-defined embedding,

$$
\psi: F B_{p_{s}}\left(\frac{1}{2}-\frac{1}{8 N^{\prime}(n)}\right) \cap F B_{p_{t}}\left(\frac{1}{2}-\frac{1}{8 N^{\prime}(n)}\right) \rightarrow F B_{p_{s}}\left(\frac{1}{2}\right) \cap F B_{p_{t}}\left(\frac{1}{2}\right) .
$$

The map $\psi$ sends fibres of $\mathscr{F}_{t}$ to fibres of $\mathscr{F}_{t}^{\prime}$, preserving affine structures and corresponding local actions. Here, the number $N^{\prime}(n)$ is as in $\S 6$. By modifying $\psi$ with the aid of a cutoff function, we obtain an $O(n)$-equivariant map (also denoted $\psi$ ) such that

(7.20.1) $\psi$ is a self-diffeomorphism of $F B_{p_{s}}\left(\frac{1}{2}\right) \cap F B_{p_{t}}\left(\frac{1}{2}\right)$.

(7.20.2) $\psi$ is the identity near the boundary.

(7.20.3) $d(\psi$, Ident $) \leq c\left(n, A, i_{0}\right) l_{j_{s}}^{i_{0}}$.

(7.20.4) $\mid \nabla^{i} \psi-$ Ident $\mid=c\left(n, A, i_{0}\right) l_{j_{s}}^{i_{0}-i}$.

(7.20.5) If $\psi\left(Z_{t}\right) \cap\left(F B_{p_{s}}\left(\frac{1}{2}-\frac{1}{4 N^{\prime}(n)}\right) \cap F B_{p_{t}}\left(\frac{1}{2}-\frac{1}{4 N^{\prime}(n)}\right)\right) \neq \varnothing$, then for some $Z_{t}^{\prime}, \psi: Z_{t} \rightarrow Z_{t}^{\prime}$, preserving the affine structure.

Let $\underline{\mathfrak{n}}_{s}, \underline{\mathfrak{n}}_{t}$ denote the sheaves of local right invariant vector fields associated to the affine structures on the fibres of $\psi\left(\mathscr{F}_{t}\right), \mathscr{F}$, as in $\S 4$. Let $\tilde{\mathfrak{n}}_{s}, \tilde{\mathfrak{n}}_{t}$ denote the associated sheaves of simply connected nilpotent Lie groups. By arguing as in $\S 4$, it is easy to check that on their common domain $\tilde{\mathfrak{n}}_{t}$ is a subsheaf of $\tilde{\mathfrak{n}}_{s}$. Then there is an obvious sheaf, $\tilde{\mathfrak{n}}_{s} \cup \tilde{\mathfrak{n}}_{t}$, over $F B_{p_{s}}\left(\frac{1}{2}-\frac{1}{4 N^{\prime}(n)}\right) \cap F B_{p_{t}}\left(\frac{1}{2}-\frac{1}{4 N^{\prime}(n)}\right)$, whose stalk at points of $F B_{p_{s}}\left(\frac{1}{2}-\frac{1}{4 N^{\prime}(n)}\right)$ coincides with $\mathfrak{n}_{s}$, and elsewhere, coincides with that of $\tilde{n}_{t}$.

Now (assuming that $\theta\left(n, A, i_{0}\right)$ is chosen sufficiently small) as in the proof of Proposition 6.1, we obtain a sheaf, $\tilde{\mathfrak{n}}^{*}$, defined all of $F M^{n}$, on which the natural action of $O(n)$ is trivial (in the sense of Proposition 4.3). This sheaf is associated to a system of (modified) maps, $f_{s}, f_{s, t}$, where $f_{s}: F B_{p_{s}}\left(\frac{1}{4}\right) \rightarrow Y_{s}^{j_{s}}$. These maps satisfy (6.1.4)-(6.1.10) (with the function $b$ as in (7.16.1) and $\lambda=\lambda(n, A))$. Moreover the affine structures on the fibres of the associated fibrations are mutually compatible.

Let $f_{s}: F B_{p_{s}}\left(\frac{1}{16}\right) \rightarrow Y_{s}^{j_{s}}$ be obtained by restriction. Denote by $\tilde{\mathfrak{n}}$ the sheaf of $F M$ gotten by applying the above construction to these maps. The sheaf $\tilde{\mathfrak{n}}$ will be shown in $\S 8$ to induce the desired structure on $M^{n}$. The fact that the domains of its defining system of maps can be enlarged to the sets $F B_{p_{s}}\left(\frac{1}{4}\right)$, is 
required in order to verify Theorems 1.3 and 1.7 .

Finally, we observe that an invariant metric close to the original one, can be constructed for our structure. Let $S^{\alpha}$ be as in (6.4.3). Recall that there are $T(n)$ of these sets. Start with those balls with centers in $S^{T(n)}$. Over each such ball, $B_{p_{s}}\left(\frac{1}{4}\right)$, construct an $\left(O(n)\right.$-invariant) invariant metric on $F B_{p_{s}}\left(\frac{1}{4}\right)$ as in $\S 4$. Using a suitable cutoff function, modify the original metric over each $F B_{p_{s}}\left(\frac{1}{4}\right) \backslash F B_{p_{s}}\left(\frac{1}{4}-\frac{1}{8 T(n)}\right)$, so as to obtain a new metric agreeing with the original one outside the union of the $F B_{p_{s}}\left(\frac{1}{4}\right)$ and with the invariant one on the union of the $F B_{p_{s}}\left(\frac{1}{4}-\frac{1}{8 T(n)}\right)$. By applying this construction successively to $S^{T(n)-1}$, $S^{T(n)-2}, \ldots$ we obtain an $O(n)$-invariant metric that is invariant, for the sheaf associated to the covering $\bigcup_{s} F B_{p_{s}}\left(\frac{1}{4}\right)$ and thus, for the sheaf $\mathfrak{n}$ as well. More precisely we get the following:

Let $\tilde{\mathfrak{n}}$ denote the metric on $F M^{n}$ induced by our original metric $g$ on $M^{n}$.

Proposition 7.21. Given $\varepsilon, i_{0}$, there exists $\theta\left(n, A, i_{0}, \varepsilon\right)$ such that by choosing $\theta=\theta\left(n, A, i_{0}, \varepsilon\right)$ in (7.16.1), we obtain a metric $\tilde{g}_{E}$ that is $O(n)$-invariant and invariant for $\mathrm{n}^{*}$, such that for $i \leq i_{0}$,

(7.21.1) $\left|\nabla^{i}\left(\tilde{g}-\tilde{g}_{\varepsilon}\right)\right| \leq \varepsilon$.

It is now routine to verify that the action of the sheaf $\mathfrak{n}$ defines a nilpotent Killing structure for the $(\rho(n, \varepsilon), 1)$-round metric, $\tilde{g}_{\varepsilon}$.

\section{THE INDUCED STRUCTURE AND METRIC ON THE BASE}

In $\S 7$ we constructed an $O(n)$-invariant Riemannian metric, $\tilde{g}_{\varepsilon}$, and an associated nilpotent Killing structure, $\tilde{\mathfrak{N}}$, on the total space, $F M^{n}$, of the frame bundle. Here, we construct the corresponding objects, $g_{\varepsilon}, \mathfrak{N}$, on the base, $M^{n}$, and show that the assertions of Theorem 1.3 and 1.7 hold. The statements of Theorems 1.3 and 1.7 are closely related and it will be convenient to prove them simultaneously. Briefly, we must

(1.3.A) construct the metric, $g_{\varepsilon}$, satisfying (1.3.1)-(1.3.3),

(1.3.B) show that $g_{\varepsilon}$ is $(\rho, k)$-round for suitable $\rho, k$ depending on $n, \varepsilon$ $((1.1 .1)-(1.1 .6))$

(1.7.A) construct the nilpotent Killing structure $\mathfrak{N}$, compatible with $g_{\varepsilon}$, and

(1.7.B) show that $\mathfrak{N}$ has compact orbits of diameter $<\varepsilon$.

Proof of Theorems 1.3 and 1.7. (1.3.A) The results of $\S \S 5-7$ were stated for $A$-regular Riemannian manifolds, with the sequence, $A$, normalized in $\S 5$. However, the metric, $g$, in Theorem 1.3 is assumed only to satisfy $|K| \leq 1$. Therefore, given $\varepsilon$ as in Theorem 1.3, we begin by replacing $g$ by the metric, $S_{\varepsilon / 2}(g)$ of Theorem 1.12. This metric is $A(n, \varepsilon / 2)$-regular. Although the sequence, $A(n, \varepsilon / 2)$, is not normalized as in $\S 5$, by an obvious scaling argument the results of $\S \S 5-7$ can still be applied to $S_{\varepsilon / 2}(g)$.

Fix $i_{0}$ (large) and $\varepsilon^{\prime}$. Starting with the metric $S_{\varepsilon / 2}(g)$, construct a metric, $\tilde{g}_{\varepsilon^{\prime}}$, on $F M^{n}$ as in Proposition 7.21. Since $\tilde{g}_{\varepsilon^{\prime}}$ is $O(n)$-invariant, there is a 
unique metric, $g_{\tau\left(\varepsilon^{\prime} \mid n\right)}$ on $M^{n}$, such that $\pi: F M^{n} \rightarrow M^{n}$ is a Riemannian submersion (see (1.13.6) for the $\tau()$ notation). Here the notation is understood to be such that if $\tau\left(\varepsilon^{\prime} \mid n\right)=\varepsilon$, then $g_{\varepsilon}$ satisfies $(1.3 .1)-(1.3 .3)$.

(1.7.B) The structure, $\tilde{\mathfrak{N}}$, on $F M^{n}$ is $O(n)$-equivariant. In particular, the action of $O(n)$ maps orbits to orbits, and hence, induces a partition of $M^{n}$ into compact submanifolds, $\{\mathscr{O}\}$. These will be seen to be the orbits of the nilpotent Killing structure on $M^{n}$. Since $\pi: F M^{n} \rightarrow M^{n}$, is distance nonincreasing, it follows that for all $\mathscr{O}$, $\operatorname{dia}(\mathscr{O})<\varepsilon$, provided that the same is true for the orbits of $\tilde{\mathfrak{N}}$. Clearly, if the constant $\theta=\theta(n, \varepsilon)$ in $\S 7$ is chosen sufficiently small, this will be the case.

(1.7.A) For the remainder of this section we will use a tilde to indicate that a point lies in the frame bundle. Let $p \in M^{n}, \tilde{p} \in \pi^{-1}(p)$ and let $Z_{\tilde{p}}$ denote the orbit of $\tilde{\mathfrak{N}}$ through $\tilde{p}$. Choose $\eta>0$ so small that

(8.1.1) $B_{\tilde{p}}(\eta)$ is simply connected,

(8.1.2) $B_{\tilde{p}}(\eta) \cap O(n)\left(B_{\tilde{p}}(\eta)\right)$ is connected,

(8.1.3) the restriction of $\tilde{\mathfrak{n}}$ to the $\eta$-tubular neighborhood, $T_{\eta}\left(Z_{\tilde{p}}\right)$, of the fibre through $\tilde{p}$ is pure,

(8.1.4) the normal injectivity radius to the orbit $\mathscr{O}_{p}$ is $\geq \eta$.

The space of local sections, $\tilde{\mathfrak{n}}\left(B_{\tilde{p}}(\eta)\right)$, is the nilpotent Lie algebra of local right invariant fields. In view of $(8.1 .1),(8.1 .2)$, it follows from Proposition 4.3 that each local vector field in $\tilde{\mathfrak{n}}\left(B_{\tilde{p}}(\eta)\right)$ is $\pi$-related to some vector field on $\pi\left(B_{\tilde{p}}(\eta)\right)=B_{p}(\eta)$. Thus we get a nilpotent Lie algebra of local Killing fields on $B_{p}(\eta)$, which, by Proposition 4.3, is independent of the choice of $\tilde{p} \in \pi^{-1}(p)$.

In a standard way, the collection of Lie algebras on the various $B_{p}(\eta)$ determines a sheaf of nilpotent Lie algebras of Killing fields on $M^{n}$. Let $\mathfrak{n}$ be the associated sheaf of simply connected Lie groups and $h$ its natural action. Obviously the orbits of this action are those considered in (1.7.B).

We now show that $\mathfrak{n}$ defines a nilpotent Killing structure.

For $\tilde{p}, \eta$ as above, we put $U=T_{\eta}\left(\mathscr{O}_{p}\right)$, where $U$ is as in Definition 1.5. Since it is clear that we can choose the same $\eta$ for all points on $\mathscr{O}_{p}$, it follows that (1.5.3) holds.

Recall that the local fibration with fibre, $Z_{\tilde{p}}$, is the restriction of a fibration defined on an open set containing $T_{1 / 8}\left(Z_{\tilde{p}}\right)$. (This fibration comes from $\tilde{\mathfrak{N}}^{*}$; see the end of $\S 7)$. Let $\mathfrak{N}^{\prime}$ denote the corresponding pure nilpotent Killing structure on $T_{1 / 8}\left(\mathscr{O}_{p}\right)$. By (8.1.3), $\mathfrak{N}^{\prime}$ extends $\mathfrak{N} \mid U$. An orbit of $\mathfrak{N}^{\prime}$ will be denoted by $\mathscr{O}^{\prime}$.

For the construction of the neighborhood, $V$, appearing in (1.5.1), (1.5.2) and in (1.3.A), we need ( $\mathfrak{N}^{\prime}$ and) Lemma 8.5 below, the statement of which requires some terminology.

Let $W$ be a length space (see [GLP]).

Definition 8.2. $B_{\underline{q}}(r) \subset W$ is star shaped if for all $w \in B_{\underline{q}}(r)$, there is a unique geodesic in $B_{\underline{q}}(r)$ joining $w$ to $\underline{q}$. 
Let $\underline{q} \in W^{l}=Y^{m} / G$, where $Y^{m}$ is a Riemannian manifold and $G$ is a compact group of isometries of $W$. Put

$$
\operatorname{An} g_{\underline{q}} W=\frac{\operatorname{Vol}\left(C_{\underline{q}} W\right)}{\operatorname{Vol}\left(S^{l-1}\right)},
$$

where $C_{\underline{q}} W$ is the set of unit vectors in the tangent cone at $\underline{q} \in W$.

Definition 8.4. $W=Y / G$ has $(\rho, k)$-bounded geometry at $w \in W$ if there exists $q \in W$ such that

(8.4.1) $\quad B_{w}(\rho) \subset B_{\underline{q}}(R) \quad$ for some star shaped $B_{\underline{q}}(R)$.

(8.4.2) $\operatorname{Ang}_{\underline{q}} W \geq \vec{k}$.

Let $\mathfrak{C} F(r, A)$ be the Hausdorff closure of the collection of $F B_{p}(r)$, such that the ball, $B_{p}(r)$, is contained in an $A$-regular manifold; compare $\S 5$. Here we do not assume that $A$ is normalized. Let $\mathfrak{C F}_{m}(r, A)$ be defined as in $\S 5$.

Lemma 8.5. For all $\delta>0$ there exists $\rho=\rho(r, A, \delta)$ and $k=k(r, A, \delta)$ such that if $Y \in \mathfrak{C F}_{m+1}(r, A)$, satisfies

(8.5.1) $d_{H}\left(Y, \mathfrak{C F}_{m}(r, A)\right) \geq \delta$,

then $Y / O(n)$ has $(\rho, k)$-bounded geometry.

Proof. This is essentially a restatement of Theorem 0.14 of [F3] (compare also (4.6.2)). It follows from Theorem 10.1 of [F3].

Let $F M^{n}$ carry the metric $\tilde{g}_{\tau\left(\varepsilon^{\prime} \mid n\right)}\left(\varepsilon^{\prime}=\varepsilon^{\prime}(\varepsilon, n)\right)$ inducing the metric $g_{\varepsilon}$ on $M^{n}$.

Fix $p \in M^{n}$. For some $Y_{s}^{j_{s}}$ as in $\S 7$, we can identify $Y_{s}^{j_{s}} / O(n)$ with the orbit space of the action of $\mathfrak{n}^{\prime}$. Let $w \in Y_{s} / O(n)$ be the projection of $p$. Let $q$ project to $\underline{q} \in Y_{s} / O(n)$ as in (8.4.1).

Remark 8.6. By way of explanation, we mention that the orbit, $\mathscr{O}_{q}^{\prime}$, should be thought of as one that has minimal dimension among all orbits in $T_{R}\left(\mathscr{O}_{q}^{\prime}\right)$.

It is an easy consequence of Lemma 8.5 and (8.4.1) that $\mathscr{O}_{q}^{\prime}$ has normal injectivity radius $\geq R=R(n, \varepsilon)$. For future reference, we note that since $M^{n}$ is $A(n, \varepsilon)$-regular, it follows that the second fundamental form, $I I_{\mathscr{O}_{q}^{\prime}}$, satisfies

$$
\left|I_{\mathscr{O}_{a}^{\prime}}\right| \leq c(n, \varepsilon) .
$$

Put $V=T_{R}\left(\mathscr{O}_{q}^{\prime}\right)$, where $V$ is the neighborhood occurring in (1.5.1), (1.5.2), and (1.1.1)-(1.1.6).

Since $V$ is a union of orbits and $\mathfrak{N}^{\prime}$ is pure, the action of $\mathfrak{n}^{\prime}$ on $V$ lifts to the action of the simply connected nilpotent Lie group, $\beta^{*}\left(\mathfrak{n}^{\prime}\right)(\widehat{V})$, on the universal covering space, $\widehat{V} \stackrel{\beta}{\longrightarrow} V$.

Let $K$ be the kernel of the action of $\beta^{*}\left(\mathfrak{n}^{\prime}\right)(\widehat{V})$ and put

$$
N_{0}=\beta^{*}\left(\mathfrak{n}^{\prime}\right)(V) / K \text {. }
$$


Apart from the assertion that $K$ is discrete (the proof of which is given in Appendix 1) it is now clear that (1.5.1), (1.5.2) hold. Modulo the proof of (1.3.B), this completes the proof of (1.7.A).

(1.3.B) Let $\tilde{q} \in \pi^{-1}(q)$. The action of $\tilde{\mathfrak{n}}^{\prime}$ on $T_{R}\left(Z_{q}^{\prime}\right)$ lifts to the action of a simply connected Lie group, $\underline{N}_{0}$, on the universal covering space, $T_{R}\left(\widehat{Z}_{\tilde{q}}^{\prime}\right)$. Moreover, it follows easily from Proposition 4.3 that the natural map, $\tau: T_{R}\left(\widehat{Z}_{\tilde{q}}^{\prime}\right) \rightarrow T_{R}\left(\mathscr{O}_{q}^{\prime}\right)$, intertwines the actions of $\underline{N}_{0}$ and $N_{0}$. Since $\pi_{1}\left(Z_{\tilde{q}}^{\prime}\right)=$ $\Lambda \subset \underline{N}_{0}$, we have $\tau(\Lambda) \subset N_{0}$. Also, by Proposition 4.3 and Lemma 8.5, $Z_{\tilde{q}}$ fibres (with a torus as fibre) over a finite covering of $\mathscr{O}_{q}$, of index at most $k$. Then the homotopy sequence for fibrations, implies that $\tau(\Lambda)$ has index at most $k$ in $\pi_{1}\left(\mathscr{O}_{q}^{\prime}\right)=\pi_{1}(V)$.

Let $H$ of $(1.1 .1)-(1.1 .6)$ be the Lie group generated by $\pi_{1}(V)$ and $N_{0}$. Now (1.1.1)-(1.1.4) and (1.1.6) are obvious.

To see $(1.1 .5)$, first note that the normal projection, $\psi: \widetilde{V} \rightarrow \widetilde{\mathscr{O}}_{q}^{\prime}$, is well defined and by (8.7) increases distances by a factor of at most $c=c(n, \varepsilon)$. If $\gamma$ is a geodesic loop on $\hat{q} \in \widehat{\mathscr{O}}_{q}^{\prime}$, then $\gamma$ is homotopic to $\underline{\gamma} \subset \widehat{\mathscr{O}}_{q}^{\prime}$ over curves of length at most $c \cdot L[\gamma]$. But since $\widehat{\mathscr{O}}_{q}^{\prime}$ is isometric to a simply connected nilpotent Lie group with left invariant metric, any closed curve in $\widehat{\mathscr{\sigma}}_{q}^{\prime}$ is contractible to a point over curves of shorter length; see Proposition 4.6.3 of [BK]. By Klingenberg's lemma on lifting homotpies inside the conjugate locus, we get

$$
L[\gamma] \geq c(n, \varepsilon) \pi .
$$

This suffices to complete the proof of (1.3.B).

Remark 8.10. The orbit space $Y / O(n)$ coincides locally with the orbit space of our structure $\mathfrak{N}$. In this connection, Lemma 8.5 expresses the fact that the orbits of our structure absorb all collapsed directions.

The following examples show that in general, the numers $\rho, k$ of Theorem 1.3 cannot be chosen independent of $\varepsilon$.

Example 8.11. We consider certain metrics on a nilmanifold $M^{3}$, viewed as the total space of a fibration, $S^{1} \rightarrow M^{3} \stackrel{\pi}{\rightarrow} T^{2}$ (with nonzero Euler class). We assume that all fibres have length $\delta^{\prime}$, that $\pi$ is a Riemannian submersion and that the metric on the base is chosen as follows. Start with a metric, $g$, which is the product of two short circles of length $\delta$. Deform $g$ slightly by introducing a small bump centered at $p$ such that the new metric, $g^{\prime}$, satisfies, $\left|K_{g^{\prime}}\right| \leq$ $1, K_{g^{\prime}}(p)=1$. Then the isometry group of $R^{2}$ equipped with the pullback metric, $\hat{g}^{\prime}$, is discrete. Moreover, for some $\eta>0$, any metric that is $\eta$-quasiisometric to $g^{\prime}$ also has this property. Thus, for the corresponding metric on $M^{3}$, the isometry group of $\widetilde{M}^{3}$, the universal covering of $M^{3}$, contains the skew product, $R \times \mathbb{Z}^{2}$ as a subgroup of finite index. Here, the center, $R$, acts by translation in the direction of the universal coverings of the $S^{1}$ fibres.

Now consider a sequence of such manifolds where $\delta^{\prime} \rightarrow 0$, while $\delta$ stays fixed, $\varepsilon=\varepsilon(\delta)$ is as in Theorem 1.3 and $\varepsilon<<\eta$. Suppose we assume that 
for this sequence and $p \in M^{3}$, we can find $\rho, k$ as in Theorem 1.3 with $\rho>\operatorname{dia}\left(T^{2}, g^{\prime}\right)$. Then neighborhood $V$ of $(1.1)$ must be $\pi^{-1}\left(T^{2}\right)=M^{3}$, and it is easy to check that for any group $H$ satisfying (1.1.1)-(1.1.6) (the definiton of $(\rho, k)$-round)) we must have $k \rightarrow \infty$ as $\delta^{\prime} \rightarrow 0$. Thus for $(\rho, k)$ as in Theorem 1.3, we must have $\rho \leq \operatorname{dia}\left(T^{2}, g^{\prime}\right)<3 \delta$, as soon as $\delta^{\prime}$ is sufficiently small.

Finally, let $\delta \rightarrow 0, \delta^{\prime} / \delta \rightarrow 0$ sufficiently fast, and choose $\varepsilon=\varepsilon(\delta)<<\eta(\delta)$ as above. Then for such a sequence if $\rho, k$ are as in Theorem 1.3, it follows that $\rho \rightarrow 0$.

On the other hand, if for each such manifold, we take $\rho=\operatorname{inj} \operatorname{rad}_{p}, V=$ $\pi^{-1}\left(B_{p}(\rho)\right)$, and $\widetilde{V}$ the universal covering of $V$, we find that we can choose $k$ as in Theorem 1.3 equal to 1 (and $H=N=R$ ).

Example 8.12. Start with flat $R^{2}$ and introduce $k$ mutually isometric tiny bumps as above centered at points with polar coordinates $\left(r, \frac{2 \pi j}{k}\right)$, where $j=0, \ldots, k-1$ and $r>0$ is a small fixed number. Then $\mathbb{Z}_{k}$ acts isometrically on $R^{2}$ by rotation about the origin. Moreover, for some $\eta>0$, any metric on $R^{2}$ that is $\eta$-quasi-isometric to the given one has at most $k$ orientation preserving isometries.

Form $R \times R^{2}$, with the product metric, where the metric on $R^{2}$ is the one just described. Let $\left(t, \Theta_{2 \pi / k}\right)$ denote the isometry of $R^{3}$ that acts by translation by $t$ units in the $R$ factor and by rotation through an angle, $2 \pi / k$, about the origin in $R^{2}$. Let $\Lambda$ be the group of isometries generated by this transformation, and put $M^{3}=\Lambda \backslash R^{3}$.

The image of the axis $(x, 0,0)$ is a circle, $S_{t}^{1} \subset M^{3}$, of length $t$. Fix $\varepsilon<\eta$ and take sufficiently small such that in particular, $t \cdot k<\varepsilon$. Then the group $N_{0}$ will be the 1-parameter group $s \rightarrow\left(s t\right.$, Ident). (Note that apart from $S_{t}^{1}$, the orbits in $M^{3}$ have length $k t$.) In this case, $\#\left(\Lambda / \Lambda \cap N_{0}\right)=k$ and $k$ can be taken arbitrarily large.

By taking products of the above manifolds with the ones in Example 8.11, we get examples for which necessarily, $\rho \rightarrow 0$, and $k \rightarrow \infty$ as $\varepsilon \rightarrow 0$.

\section{APPENDIX 1. LOCAL STRUCTURE OF MANIFOLDS OF BOUNDED CURVATURE}

The proof of Theorems 1.3 and 1.7 given in $\S 8$ has as a consequence that every point $p \in M^{n}$ is contained in a neighborhood of the form $T_{R}\left(\mathscr{O}_{q}^{\prime}\right)$, for the metric $g_{\varepsilon}$. We now give a more explicit desription of the metric structure of $T_{R}\left(\mathscr{O}_{q}^{\prime}\right)$.

First of all, examination of the proof of Theorem 10.1 of [F3] shows that given $R_{2}$ there exists $R_{1}\left(n, \varepsilon, R_{2}\right)$ such that we can choose $R_{1}\left(n, \varepsilon, R_{2}\right) \leq$ $R \leq R_{2}$, provided we take $\rho=\rho\left(n, \varepsilon, R_{2}\right)$ sufficiently small and $k=$ $k\left(n, \varepsilon, R_{2}\right)$ sufficiently large.

For $R_{2}=R_{2}(n, \varepsilon)$ sufficiently small, we can replace $g_{\varepsilon} \mid T_{R}\left(\mathscr{O}_{q}^{\prime}\right)$ by the natural metric on the tube of radius $R$ in the normal bundle, $\nu\left(\mathscr{O}_{q}^{\prime}\right)$, and obtain a metric that satisfies (1.3.1)-(1.3.3) (with $\varepsilon$ replaced by $2 \varepsilon$ ). Here, we identify 
$T_{R}\left(\mathscr{O}_{q}^{\prime}\right)$ and this tube via the normal exponential map. Relations (1.3.1)-(1.3.3) continue to hold for the new metric (and $2 \varepsilon$ ), provided we take

(A1.1.1) $\rho=\rho\left(n, \varepsilon, R_{2}(n, \varepsilon)\right)$

(A1.1.2) $k=k\left(n, \varepsilon, R_{2}(n, \varepsilon)\right)$,

(which still depend only on $n, \varepsilon$ ). In this way, we obtain a more canonical local model for the geometry.

The bundle $\nu\left(\mathscr{O}_{q}^{\prime}\right)$ can be described up to isometry as follows.

It follows easily from (4.1) that there is a principal bundle,

$$
C^{\prime} \rightarrow Z_{\tilde{q}} \rightarrow \mathscr{O}_{q}^{\prime}
$$

Here $Z_{\tilde{q}}$ is isometric to the almost flat manifold $\Lambda \backslash N$, with some left invariant metric, and $\Lambda \subset N_{L}$. The group $C^{\prime} \subset O(n)$, is the isotropy group of $Z_{\tilde{q}}$. It satisfies

$$
e \rightarrow(\Lambda \cap C) \backslash C \rightarrow C^{\prime} \rightarrow B \rightarrow e
$$

where $C$ is contained in the center of $\mathfrak{n},(\Lambda \cap C) \backslash C$ is a torus and $B$ is a finite group of order $\leq k$.

Since the local fibration, $Z \rightarrow F M \stackrel{f_{s}}{\longrightarrow} Y_{s}$ is a Riemannian submersion for the metric, $\tilde{g}_{\varepsilon}$, the normal bundle to any fibre has a natural isometric trivialization, $\nu\left(Z_{\tilde{q}}\right)=Z_{q}^{n-j} \times R^{j}$. The action of $O(n)$ preserves the fibration $f_{s}$. Thus, the action of $C$ on $\nu\left(Z_{\tilde{q}}\right)$ is of the form

$$
g(x, y)=(g x, \phi(y)),
$$

where $g x$ denotes the natural action of $C$ on $Z_{\tilde{q}}$ and $\phi: C \rightarrow O(j)$ is an orthogonal representation of $C$. The representation $\phi$ preserves the splitting, $R^{j}=R^{m} \oplus R^{j-m}$, where $R^{m}$ is the subspace orthogonal to both the fibre, $Z_{\tilde{q}}$, and the fibres of the princpal bundle (i.e., $O(n)$ orbits). The following proposition is then obvious.

Proposition A1.5. The bundle $\nu\left(\mathscr{O}_{q}^{\prime}\right)$, with its natural metric, is isometric to the vector bundle (with its natural metric) associated to the principal fibration, (A1.2), via the representation $\phi: C^{\prime} \rightarrow O(m)$.

We now give a relation between the algebraic structure of the stalk, $\mathfrak{n}_{p}$, of our $N$-structure and the volume of the ball, $B_{p}(\rho)$.

Given a Lie algebra $\mathfrak{n}$, let $\mathfrak{n}=\mathfrak{n}_{0} \supset[\mathfrak{n}, \mathfrak{n}]=\mathfrak{n}_{1} \supset \cdots$ be its lower central series. Put

$$
d(\mathfrak{n})=\sum \operatorname{dim} \mathfrak{n}_{k} .
$$

Theorem A1.7. There exists a constant $C=C(n, \rho, k)$ such that, if $\mathfrak{n}$ is an $N$ structure on $M$ and $g$ is an $\mathfrak{n}$-invariant $(\rho, k)$-round metric as in Theorem 1.7, then

$$
\text { (A1.7.1) } \operatorname{Vol}\left(B_{p}(\rho)\right) \leq C \varepsilon^{d\left(\mathfrak{n}_{p}\right)} \text {. }
$$

Corollary A1.8. For each $C$ there exists $\varepsilon_{0}$ such that if in the situation of Theorem A1.7,

(A1.8.1) $\operatorname{Vol}\left(B_{p}(\rho)\right) \geq C \varepsilon^{\operatorname{dim} n_{p}}$,

for $\varepsilon<\varepsilon_{0}$, then $\mathfrak{n}_{p}$ is Abelian. 
Remark A1.9.

(A1.9.1) $\quad \operatorname{Vol}\left(B_{p}(\rho)\right) \geq C \varepsilon^{d\left(\mathfrak{n}_{p}\right)}$,

does not hold in general. The flat metric $\varepsilon^{2} d x^{2}+\varepsilon^{4} d y^{2}$ on $T^{2}$ is a counterexample.

Remark A1.10. We say that $\mathfrak{n}$ is filtered if there exists $\mathfrak{n}_{(i)} \subset \mathfrak{n}$ such that $\left[\mathfrak{n}_{(i)}, \mathfrak{n}_{(j)}\right] \subset \mathfrak{n}_{(i+j)}$. Put $d^{\prime}(\mathfrak{n})=\sum \operatorname{dim} \mathfrak{n}_{(i)}$. Then there exists a locally homogeneous metric on $M=\Lambda \backslash N$ such that

$(\mathrm{A} 1.10 .1) \operatorname{Vol}\left(B_{p}(\rho)\right) \sim C \varepsilon^{d^{\prime}\left(\mathfrak{n}_{p}\right)}$.

Proof of Theorem A1.7. We replace $B_{p}(\rho)$ by a normal covering space $\widetilde{F B}_{p}(\rho)$ of $F B_{p}(\rho)$, of order $\leq k$. Then, in view of the local description of the metric given at the beginning of this appendix, it suffices to show the following

Lemma A1.11. There exist $\varepsilon_{n}$ and $C_{n}$ such that if $\varepsilon<\varepsilon_{n}$ then the following holds: Let $(\Lambda \backslash N, \bar{g})$ be a compact $n$-dimensional nilmanifold equipped with a locally homogeneous metric. Suppose

(A1.11.1) $\operatorname{dia}(\Lambda \backslash N, \bar{g})<\varepsilon$,

(A1.11.2) $\left|K_{(\Lambda \backslash N, \bar{g})}\right|<1$;

then we have

(A1.11.3) $\operatorname{Vol}(\Lambda \backslash N)<C_{n} \varepsilon^{d(n)}$,

where $\mathfrak{n}$ is the Lie algebra of $N$.

Proof. Let $\Lambda_{1}=[\Lambda, \Lambda] \supset \cdots \supset \Lambda_{k+1}=\left[\Lambda, \Lambda_{k}\right] \cdots$. By [BK, 2.4.2], there exists $C$ such that $\Lambda_{k}$ is generated by homotopy classes of loops whose length is smaller than $\varepsilon^{k}$.

Let $N_{0} \supset \cdots \supset N_{k} \cdots$ be the lower central series of $N$. Suppose $N_{k} \neq 1$, $N_{k+1}=1$. Since $\Lambda_{k} \backslash N_{k}$ is flat and since its fundamental group is generated by loops whose length is smaller than $\varepsilon^{k}$, it follows that

(A1.12.1) $\operatorname{Vol}\left(\Lambda_{k} \backslash N_{k}\right)<C \varepsilon^{k \operatorname{dim} N_{k}}$.

Similarly we have

$(\mathrm{A} 1.12 .2) \operatorname{Vol}\left(\Lambda_{k-1} \backslash N_{k-1} / N_{k}\right)<C \varepsilon^{(k-1)\left(\operatorname{dim} N_{k-1}-\operatorname{dim} N_{k}\right)}$.

Therefore

(A1.12.3) $\operatorname{Vol}\left(\Lambda_{k-1} \backslash N_{k-1}\right)<C \varepsilon^{\operatorname{dim} N_{k}+(k-1) \operatorname{dim} N_{k-1}}$.

Inductively we have

(A1.12.4) $\operatorname{Vol}\left(\Lambda_{k-i} \backslash N_{k-i}\right)<C \varepsilon^{\Sigma_{j=0}^{i-1} \operatorname{dim} N_{k-j}+(k-i) \operatorname{dim} N_{k-i}}$.

The lemma follows immediately.

Remark A1.13. The construction in [FY, §7] is closely related to the lemma. 
Lastly, we return to the point in the proof of Theorem 1.7 whose proof was deferred. Namely, we show that the group $K$ in (8.8) is discrete. For this it suffices to show the following. Let $M^{n}, \varepsilon$ be as in Theorem 1.7. Let $p \in M^{n}$, $\tilde{p} \in \pi^{-1}(p)$ and let $Z_{\tilde{p}}$ be the corresponding orbit of $\tilde{\mathfrak{n}}$.

Proposition A1.14. If $\varepsilon<\varepsilon(n)$ is sufficiently small, then for all $p \in M^{n}$ and $\delta>0$, there exists $p_{1} \in M^{n}$ such that $d\left(p, p_{1}\right)<\delta$ and $\operatorname{dim} \mathscr{O}_{p_{1}}=\operatorname{dim} Z_{\tilde{p}}$.

Proof. The identity component, $C$, of $C^{\prime} \subset O(n)$, the isotropy group of $Z_{\tilde{p}}$, is a torus whose Lie algebra lies in the center of $\tilde{\mathfrak{n}}$. If $\operatorname{dim} C=m$, then

$$
\operatorname{dim} Z_{\tilde{p}}-m=\operatorname{dim} \mathscr{O}_{p} .
$$

The torus $C$ acts on a covering space $\widehat{T}_{\eta}\left(\mathscr{O}_{p}\right)$, of order at most $k$, by rotation in the fibres normal to the lifted orbit, $\widehat{\mathscr{O}}_{p}$. If this action is effective, its principal orbits have dimension $m$. It follows easily that if $p_{1}$ lies on a principal orbit sufficiently close to $p$, then

$$
\operatorname{dim}\left(\mathscr{O}_{p_{1}}\right)=\operatorname{dim} Z_{\tilde{p}}
$$

To see that the action of $C$ (or equivalently, that of $\mathfrak{n}$ ) is effective, recall the following standard fact.

Lemma A1.17. There exists $c_{1}(n, \varepsilon), c_{2}(n, \varepsilon)$ such that the map of pseudogroups, $\pi_{*}: \pi_{1}\left(F M, \tilde{p}, c_{1}\right) \rightarrow \pi_{1}\left(M, p, c_{2}\right)$, is an injection.

As a consequence of Lemma A1.17, the unique inverse to the map $\pi_{*}$ is gotten by lifting elements of $\pi_{1}\left(M, p, c_{2}\right)$ via their differentials, to obtain elements of $\pi_{1}\left(F M, \tilde{p}, c_{1}\right)$.

Put $\pi_{*}^{-1}(h)=\tilde{h}$. Let $d($,$) denote the uniform norm. Clearly, there exists$ $c(n)>0$, such that for all $\tilde{h} \in \pi_{1}\left(F M, \tilde{p}, c_{1}\right)$,

$$
d(\tilde{h}, \text { Ident }) \leq c(n) d(h, \text { Ident }) .
$$

(Recall that a local isometry that fixes a point and a frame at that point is the identity map.)

Now identify $\tilde{\mathfrak{n}}_{\tilde{p}} \quad$ with the universal covering, $\hat{Z}_{\tilde{p}}$, of $Z_{\tilde{p}}$. Then we can regard $\pi_{1}\left(F M, \tilde{p}, c_{1}\right) \subset \Lambda$. Since $\Lambda$ is $b\left(i_{j_{s}}\right)$-dense in $\widehat{Z}_{\tilde{p}}$, it follows with the help of (4.7) that an estimate like (A1.18) holds for all elements of $\tilde{\mathfrak{n}}_{p}$ (possibly with a different constant $c(n)$ ). This suffices to complete the proof.

Remark A1.19. In case $M^{n}$ itself is almost flat, the statement of Proposition A1.14 yields a first main step in the proof of the theorem on almost flat manifolds; i.e., short loops with not too big holonomy have holonomy at most comparable to their lengths; compare [Gh2]. This argument might appear to be circular since it depends on constructing a fibration of the frame bundle with nilmanifold fibres. However, in this case the first step of the almost flat theorem 
is trivial since a short loop in the frame bundle automatically has holonomy at most of size comparable to its length.

\section{APPENDIX 2. FIBRATION ISOTOPY}

In this appendix we give a version of a well-known result to the effect that if two fibrations are sufficiently $C^{1}$-close, then one of them can be deformed onto the other. For the applications in $\S 6$ and $\S 7$, it is important that the required degree of closeness is independent of the injectivity radius of the total space and of the Hessian of one of the projection maps.

Let $X, Y$ be $A$-regular Riemannian manifolds with $A_{0}=1$, on which a compact group, $G$, acts by isometries. Assume that for all $y \in Y$,

(A2.1) inj $\operatorname{rad}_{y} \geq \min (l, d(y, \partial Y))$.

(Here and below we use the notation of (2.2), (2.3).)

Proposition A2.2. Let $f, g: X \rightarrow Y$ be G-equivariant maps for which the following hold:

(A2.2.1) $f$ is a 1-almost Riemannian submersion.

(A2.2.2) $f$ is $\left\{B_{i} l^{1-i}\right\}$-regular.

(A2.2.3) $g$ is $C$-regular.

(A2.2.4) For some $\eta>0, f, g$ are $\varepsilon$-close in the $C^{1}$-topology, with $\varepsilon(\imath \eta)^{-1}<$ $\beta_{0}\left(B_{1}, B_{2}\right)$, sufficiently small.

Then there exists a G-equivariant self-diffeomorphism, $\psi$, of $X$, such that

(A2.2.5) $f=g \psi$ on $X_{\eta}$.

(A2.2.6) $\psi$ is the identity near $\partial X$.

(A2.2.7) $\psi$ is $c\left(n, B_{0}, B_{1}\right) \varepsilon$ close to the identity map in the $C^{1}$-topology.

(A2.2.8) $\psi$ is $\left\{D_{i}(A, B, C)(\eta l)^{1-i}\right\}$-regular. Moreover, $D_{i}$ depends only on $B_{k}, C_{k}, k \leq i$ (and finitely many $A_{i}$ ).

Proof. By scaling the metrics on $X$ and $Y$ we can assume $l, \eta \geq 1$.

By a standard computation, for all $y \in Y$,

(A2.3.1) $\left|I I_{f^{-1}(y)}\right| \leq c_{1}\left(B_{2}\right)$.

Moreover, for $y \in Y_{1}$, the normal injectivity radius, $l$, of $f^{-1}(y)$, satisfies (A2.3.2) $l \geq c_{2}\left(B_{2}\right)$.

Indeed, the normal exponential map is nonsingular on a tube of radius $c_{3}\left(B_{2}\right)$. Thus, by a standard argument, if $l<c_{2}\left(B_{2}\right)$ there exists a geodesic segment, $\gamma$, of length $2 l$, with $\gamma(0), \gamma(2 l) \in f^{-1}(y)$ and $\gamma^{\prime}(0), \gamma^{\prime}(2 l)$ normal to $f^{-1}(y)$. For $l$ sufficiently small relative to $c_{1}, \gamma^{\prime}(t)$ is almost normal to the fibre through $\gamma(t)$, for all $0 \leq t \leq 2 l$. It follows that $f(\gamma)$ is a short loop on $y$ of small geodesic curvature. For $l$ sufficiently small this contradicts inj rad $y \geq 1$.

Note that all fibres $f^{-1}(y), g^{-1}(y)$ are $c \varepsilon$-close, together with their tangent planes. For $\varepsilon$-sufficiently small, normal projection from $g^{-1}(y)$ to $f^{-1}(y)$ is a covering map. Moreover an argument like that of the previous paragraph shows 
that this map is actually a diffeomorphism. The collection of all such maps defines a $G$-equivariant map $\hat{\psi}$ with domain say, $X_{1 / 2}$, satisfying (A2.2.5). Let $\chi$ be a function such that

(A2.4.1) $\chi \mid X_{1} \equiv 1$,

(A2.4.2) $\chi \mid X \backslash X_{1 / 2} \equiv 0$,

constructed from a $G$-equivariant smoothing of the distance function as in $\S 2$. For $\varepsilon, \beta_{0}$ as in (A2.2.4), sufficiently small, put

(A2.4.3) $\psi=\chi \hat{\psi}+(1-\chi)$ Ident.

Then, $\psi$ is easily seen to satisfy $(\mathrm{A} 2.2 .6)-(\mathrm{A} 2.2 .8)$.

\section{ACKNOWLEDGMENT}

We are grateful to Z. Shen and G. Wei for helpful comments.

\section{REFERENCES}

[A] U. Abresch, Über das glatten Riemannisher metriken, Habilitationsschrift, Reinischen Friedrich-Willhelms-Universität Bonn, 1988.

[Ba] S. Bando, Real analyticity of solutions of Hamilton's equation, Math. Z. 195 (1987), 93-97.

[BMR] J. Bemelmans, Min-Oo, and A. Ruh, Smoothing Riemannian metrics, Math. Z. 188 (1984), 69-74.

[BK] P. Buser and H. Karcher, Gromov's almost flat manifolds, Asterisque 81 (1981), 1-148.

[C] J. Cheeger, Finiteness theorems of Riemannian manifolds, Amer. J. Math. 92 (1970), 61-74.

[CG1] J. Cheeger and M. Gromov, On the characteristic numbers of complete manifolds of bounded curvature and finite volume, Differential geometry and complex analysis, H. E. Rauch Memorial Volume, Springer-Verlag, Berlin, 1985.

[CG2] __ Bounds on the von Neumann dimension of $L^{2}$-cohomology and the Gauss-Bonnet theorem for open manifolds, J. Differential Geom. 21 (1985), 1-31.

[CG3] _ Collapsing Riemannian manifolds while keeping their curvature bounded I, J. Differential Geom. 23 (1986), 309-346.

[CG4] __ Collapsing Riemannian manifolds while keeping their curvature bounded II, J. Differential Geom. 32 (1990), 269-298.

[F1] K. Fukaya, Collapsing Riemannian manifolds to ones of lower dimension, J. Differential Geom. 25 (1987), 139-156.

[F2] _ Collapsing Riemannian manifolds to ones of lower dimension II, J. Math. Soc. Japan 41 (1989), 333-356.

[F3] _ A boundary of the set of the Riemannian manifolds with bounded curvatures and diameters, J. Differential Geom. 28 (1988), 1-21.

[F4] _ Hausdorff convergence of Riemannian manifolds and its applications, Recent Topics in Differential and Analytic Geometry (T. Ochiai, ed.), Kinokuniya, Tokyo, 1990.

[F5] _ A compactness of a set of aspherical Riemannian orbifolds, A Fete of Topology, Tamura Memorial Volume, Academic Press, Boston, MA, 1988, pp. 391-413.

[FY] K. Fukaya and T. Yamaguchi, Almost nonpositively curved manifolds, J. Diferential Geom. 33 (1991), 69-90.

[Gh1] P. Ghanaat, Almost Lie groups of Type $\mathbf{R}^{n}$, J. Reine Angew. Math. 401 (1989), 60-81.

[Gh2] __ Geometric construction of holonomy coverings for almost flat manifolds, J. Differential Geom. 34 (1991), 571-579. 
[GhMR] P. Ghanaat, M. Min-oo, and E. Rhu, Local structure of Riemannian manifolds, Indiana Univ. Math. J. 39 (1990), 1305-1312.

[G1] M. Gromov, Almost flat manifolds, J. Differential Geom. 13 (1978), 231-241.

[G2] __ Volume and bounded cohomology, Publ. Math. I.H.E.S. 56 (1983), 213-307.

[GLP] M. Gromov (rédigé par J. Lafontaine and P. Pansu), Structure métrique pour les variétes riemannienne, Cedic Fernand Nathan, Paris, 1987.

[GrK] K. Grove and H. Karcher, How to conjugate $C^{1}$ close actions, Math. Z. 132 (1973), 11-20.

[GW] R. Greene and H. Wu, Lipschitz convergence of Riemannian manifolds, Pacific J. Math. 131 (1988), 119-141.

[K] H. Karcher, Riemannian center of mass and molifier smoothing, Comm. Pure Appl. Math. 30 (1977), 509-541.

[P] S. Peters, Convergence of Riemannian manifolds, Comp. Math. 62 (1987), 3-16.

[R] E. Ruh, Almost flat manifolds, J. Differential Geom. 17 (1982), 1-14.

[Rag] M. S. Raghunathan, Discrete subgroups of Lie group, Springer-Verlag, Berlin, 1972.

[Shi] W.-X. Shi, Deforming the metric on complete Riemannian manifolds, J. Differential Geom. 30 (1989), 225-301.

[Y] T. Yamaguchi, Collapsing and pinching in lower curvature bound, Ann. of Math. (2) 133 (1991), 317-357.

Department of Mathematics, New York University, Courant Institute of Mathematical Sciences, New York, New York 10012

Department of Mathematics, Faculty of Science, University of Tokyo, Hongo, Tokyo, JAPAN 113

Institute des Hautes Etudes SCientifiques, (91440) Bures-SUR-Yvette, France 